OPEN ACCESS

Edited by:

Abdur Rauf,

University of Swabi, Pakistan

Reviewed by:

Abdul Wadood,

Abdul Wali Khan University Mardan,

Pakistan

Steven Fiering,

Dartmouth College, United States

*Correspondence:

Feng Yan

yan_feng@scu.edu.cn

Yulan Peng

yulanpeng@163.com

Specialty section:

This article was submitted to Experimental Pharmacology and Drug

Discovery,

a section of the journa

Frontiers in Pharmacology

Received: 10 August 2021

Accepted: 15 October 2021

Published: 15 November 2021

Citation:

Zhang $H, X u$ J, Gao B, Wang H, Huang J, Zhou J, Yang R, Yan F and

Peng Y (2021) Synergistic Cascade

Strategy Based on Modifying Tumor

Microenvironment for Enhanced

Breast Cancer Therapy.

Front. Pharmacol. 12:750847.

doi: 10.3389/fphar.2021.750847

\section{Synergistic Cascade Strategy Based on Modifying Tumor Microenvironment for Enhanced Breast Cancer Therapy}

Huan Zhang ${ }^{1}$, Jinshun $\mathrm{Xu}^{1}$, Binyang Gao ${ }^{1}$, Hong Wang ${ }^{1}$, Jianbo Huang ${ }^{2}$, Jie Zhou ${ }^{1}$, Rui Yang ${ }^{1}$, Feng Yan $^{2 *}$ and Yulan Peng ${ }^{1 *}$

${ }^{1}$ Department of UItrasound, West China Hospital, Sichuan University, Chengdu, China, ${ }^{2}$ Laboratory of Ultrasound Imaging Drug, West China Hospital, Sichuan University, Chengdu, China

Background: Triple-negative breast cancer (TNBC) is the most aggressive subtype of breast cancer with very few treatment options. Although tumor-targeted nanomedicines hold great promise for the treatment of TNBC, the tumor microenvironment (TME) continues to be a major cause of failure in nanotherapy and immunotherapy. To overcome this barrier, we designed a new synergistic cascade strategy (SCS) that uses mild hyperthermia and smart drug delivery system (SDDS) to alter TME resistance in order to improve drug delivery and therapeutic efficacy of TNBC.

Methods: Mild hyperthermia was produced by microwave (MW) irradiation. SDDS were formulated with thermosensitive polymer-lipid nanoparticles (HA-BNPs@Ptx), composed of polymer PLGA, phospholipid DPPC, hyaluronic acid (HA, a differentiation-44-targeted molecule, also known as CD44), 1-butyl-3-methylimidazolium-L-lactate (BML, a MW sensitizer), and paclitaxel (Ptx, chemotherapy drug). 4T1 breast tumor-bearing mice were treated with two-step MW combined with HA-BNPs@Ptx. Tumors in mice were pretreated with first MW irradiation prior to nanoparticle injection to modify and promote TME and promoting nanoparticle uptake and retention. The second MW irradiation was performed on the tumor $24 \mathrm{~h}$ after the injection of HA-BNPs@Ptx to produce a synergistic cascade effect through activating BML, thus, enhancing a hyperthermia effect, and instantly releasing Ptx at the tumor site.

Results: Multifunctional CD44-targeted nanoparticles HA-BNPs@Ptx were successfully prepared and validated in vitro. After the first MW irradiation of tumors in mice, the intratumoral perfusion increased by two times, and the nanoparticle uptake was augmented by seven times. With the second MW irradiation, remarkable antitumor effects were obtained with the inhibition rate up to $88 \%$. In addition, immunohistochemical analysis showed that SCS therapy could not only promote tumor cell apoptosis but also significantly reduce lung metastasis.

Conclusion: The SCS using mild hyperthermia combined with SDDS can significantly improve the efficacy of TNBC treatment in mice by modifying TME and hyperthermiamediated EPR effects.

Keywords: breast cancer, tumor microenvironment, drug delivery, hyperthermia, nanoparticles 


\section{INTRODUCTION}

Breast cancer is the most common cancer and one of the leading causes of cancer-related death in women worldwide (Tajbakhsh et al., 2018). Despite the early detection and intervention, metastatic breast cancers remain largely incurable, especially triple-negative breast cancer (TNBC) (Mu et al., 2017; Thakur and Kutty, 2019). TNBC is an aggressive subtype of breast cancer and accounts up to $10 \%-20 \%$ of all breast cancer cases (Ding et al., 2021). Due to the lack of specific targets and high probability of metastasis, currently available treatment options are very limited (Hu et al., 2020). Metastatic breast cancer is characterized by a unique tumor microenvironment (TME), which differs from other subtypes. The components of TME, including transformed extracellular matrix (ECM), soluble factors, immune suppressive cells, epigenetic modifications, and re-programmed fibroblasts, together hamper antitumor response and help in the progression and metastasis of TNBC (Junttila and de Sauvage, 2013; Deepak et al., 2020). Another barrier to breast cancer is its high heterogeneity, which complicates treatment (Pashayan et al., 2020; Wang et al., 2020). For example, a small piece of tumor tissue obtained by biopsy does not necessarily represent all the tumor components (Januškevičienè and Petrikaite, 2019). In addition, high interstitial fluid pressure (IFP) generated by TME also severely limits drug delivery to tumor cells, especially in immunotherapy and nanotherapy (Yang and Gao, 2017). High IFP in TME may compress blood vessels, resulting in reduced intratumoral blood flow and nanodrug delivery (Griffon-Etienne et al., 1999; Nia et al., 2020). Since the TME is involved in the proliferation, angiogenesis, apoptosis inhibition, immune system suppression, and drug resistance of metastatic breast cancer, it becomes recently an important target of TNBC therapy (Nienhuis et al., 2015; Lang et al., 2019; Feng et al., 2020).

Hyperthermia combined with nanoparticles loaded with chemotherapeutic agents holds great promise for the treatment of cancer (Dunne et al., 2020). However, the efficacy in solid cancer treatment has not been clinically proven. For example, numerous clinical trials have been conducted since 2006 using ThermoDox, doxorubicin-loaded low temperature-sensitive liposomes (LTSLs), to treat hepatic, colorectal, prostate, and breast cancer (May and Li, 2013). Hyperthermia can be achieved using different heating techniques, such as radiofrequency (RF), focused ultrasound (FUS), and microwave (MW) (Markezana et al., 2020). A phase I study (TARDOX) recently showed that the combined treatment of LTSLs and non-invasive FUS hyperthermia seems to be clinically feasible, safe, and able to enhance intratumoral drug delivery (Lyon et al., 2018). Although an increased intratumoral drug delivery has been demonstrated in a preclinical study, most of phase II and phase III trials of ThermoDox failed to demonstrate the benefit of the combined treatment over chemo- or thermal therapy alone (Tak et al., 2018; De Maar et al., 2020). Therefore, the preclinical research and clinical translation of thermosensitive nanomedicines are still facing huge demands and challenges (Dou et al., 2017; Nardecchia et al., 2019).
Hyperthermia induced by MW is a promising adjuvant therapy, which can be used to induce the apoptosis of tumor cells and destroy ECM in TME (Paulides et al., 2020). Several techniques have been used to generate local heating for tumor repression, including nearinfrared photothermal therapy, magnetic thermal therapy, RF thermal therapy, ultrasonic hyperthermia, and microwave thermal therapy. The MW thermal therapy of tumors has attracted much interest recently, due to the maneuverability, faster heat generation, depth of penetration in tissues, and perfect ability of killing tumor cells (Dunne et al., 2020; Paulides et al., 2020). MW can penetrate deeply into most of solid tumors in patients, does not produce drug resistance of TME, and has no systemic side effects (Wu et al., 2019). Mild MW hyperthermia can also induce apoptosis of tumor cells at $39^{\circ}-45^{\circ} \mathrm{C}$ (Qi et al., 2019; Dunne et al., 2020). However, there are very few reports using MW hyperthermia combined with Ptx-loaded nanoparticles to treat metastatic breast cancers. Therefore, the need for combination therapy to overcome the resistance of breast tumors and improve the efficacy of current treatment is very clear.

In the present study, we proposed a new approach to enhance the bioavailability of nanodrugs and the efficacy of hyperthermia to treat triple negative breast cancer called "Synergistic Cascade Strategy" (SCS). The strategy consists of using local MW hyperthermia combined with smart drug delivery system (SDDS) to treat TNBC in a $4 \mathrm{~T} 1$ breast cancer-bearing mice model. SDDS was formulated with polymer-lipid nanoparticles (HA-BNPs@Ptx), composed of polymer PLGA, phospholipid DPPC, hyaluronic acid (HA, targeting differentiation-44, also known as CD44), 1-butyl-3-methylimidazolium-L-lactate (BML, a MW sensitizer), and paclitaxel (Ptx, chemotherapy drug). Our previous work (Xu et al., 2019a) showed that MW-responsive nanoplatform made of lipid nanoparticles, containing DPPC and BML, could efficiently deliver doxorubicin and inhibit hepatocellular carcinoma progression with distant lung metastasis. The rationale of SCS is the following: (Tajbakhsh et al., 2018) performing the first MW on tumor to induce mild hyperthermia $\left(\sim 43^{\circ} \mathrm{C}\right)$, in order to reduce IFP in TME and increase intratumoral blood flow. The mild hyperthermia also allows "permeabilizing" tumoral vessels and alter the tumor surrounding matrix, thus, promoting the extravagation and penetration of nanoparticles to reach tumor cells (Thakur and Kutty, 2019); injecting long circulating target PLGA-DPPC nanoparticles immediately following the first MW exposure to allow the maximal accumulation of nanoparticles at tumor sites (Mu et al., 2017); and after nanoparticle accumulation, performing second MW exposure to activate the sensitizer BML, thus, increasing rapidly the temperature inside the tumor and instantly release Ptx from nanoparticles to induce tumor cell apoptosis and destroy the ECM in TME, though there are synergistic cascade effects of hyperthermia and chemotherapy agents. Figure 1 illustrates the scenario of the above approach.

\section{MATERIALS AND METHODS}

\section{Materials}

$\mathrm{NH}_{2}$-PLGA- $\mathrm{NH}_{2}$ (lactide:glycolide, 50/50, MW: $8 \mathrm{kDa}$ ) was obtained from Xi'an Rui-Xi Biological Technology Co. Ltd. 


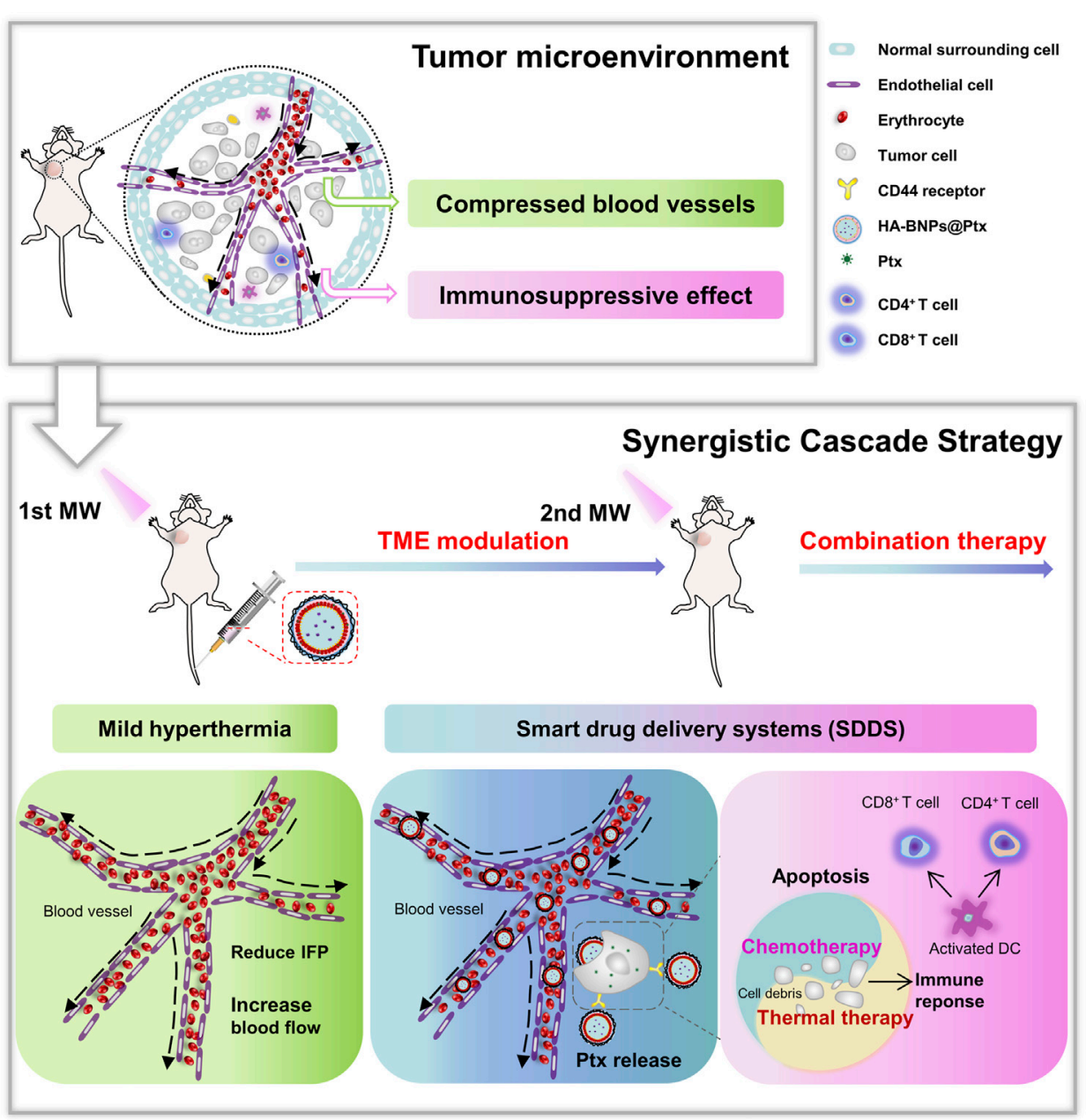

FIGURE 1 | lllustration of tumor microenvironment and treatment process with synergistic cascade strategy.

(Xi'an, Shaan Xi, China). 1-Butyl-3-methylimidazolium-L-lactate (BML, MW: 228.29 Da) was provided by Cheng Jie Chemical Co. Ltd. (Shanghai, China). Hyaluronic acid (HA, MW: $3.8 \mathrm{kDa}$ ) was purchased from Dalian Meilun Biotechnology Co. Ltd. (Dalian, Liao Ning, China). Paclitaxel (Ptx, MW: 853.93 Da) was purchased from Shanghai Aladdin Biotechnology Co. Ltd. (Shanghai, China). 1,2-Dipalmitoyl-sn-glycerol-3phosphocholine (DPPC, MW: 734.1 Da), polyvinyl alcohol (PVA, MW: $30-70 \mathrm{kDa})$, 2-(N-morpholino) ethane sulfonic acid (MES), N-hydroxysuccinimide (NHS), and 1-ethyl-3-(3dimethylaminopropyl) carbodiimide (EDC) were obtained from Sigma-Aldrich Corporation (St. Louis, MO, USA). All reagents used in this work were of analytical grade without further purification.

\section{Preparation of HA-BNPs@Ptx}

The double emulsification method was used to synthesize Ptx and BML-loaded DPPC-PLGA hybrid nanoparticles (BNPs@Ptx) following a previous report (Xu et al., 2019b). Briefly, $10 \mathrm{mg}$ of DPPC, $20 \mathrm{mg}$ of $\mathrm{NH}_{2}-\mathrm{PLGA}-\mathrm{NH}_{2}$, and $2 \mathrm{mg}$ of Ptx were thoroughly dissolved in $2 \mathrm{ml}$ of dichloromethane (DCM). BML, $400 \mu \mathrm{l}(0.5 \mathrm{mg} / \mathrm{ml}$, in deionized water $)$, was mixed in the solution. The Ptx and BML solution was emulsified in an ice bath ( 2 min, with a 5-s on-off duty cycle) using an ultrasonic oscillation instrument (SCIENTZ-IID, Ningbo Scientz Biotechnology Co., Ltd., Ningbo, Zhejiang, China) at $12 \%$ power $(24 \mathrm{kHz}, 600 \mathrm{~W})$. PVA, $8 \mathrm{ml}(2 \% \mathrm{w} / \mathrm{w}$, in water $)$ solution, was further added to the emulsion and emulsified as before. DCM was then evaporated in a fume hood for $3 \mathrm{~h}$ under magnetic stirring. Finally, BNPs@Ptx was collected by centrifugation at $10,000 \times g$ for $10 \mathrm{~min}$ and washed twice with double distilled water. Nanoparticles targeting CD44 were prepared by covalently coupling hyaluronic acid to BNPs@Ptx via amino groups on PLGA (Alam et al., 2017). Briefly, $2 \mathrm{mg}$ of HA was dissolved in $195 \mathrm{mg}$ of MES buffer $(\mathrm{pH}=5.5)$. EDC $(27 \mathrm{mg}$ ) and $8.6 \mathrm{mg}$ of NHS were then added to the HA solution. Subsequently, the mixture was incubated with $1 \mathrm{ml}$ of BNPs@Ptx suspension under continuous stirring for $24 \mathrm{~h}$ on ice bath. HABNPs@Ptx was collected and purified by centrifugation and washed with double distilled water two times. 


\section{Characterization of HA-BNPs@Ptx Morphology and physicochemical properties of HA-BNPs@Ptx}

Transmission electron microscopy (TEM, Tecnai G2 F30, FEI Co., Ltd., Hillsboro, OR, USA) and scanning electron microscope (SEM, SU8020, Hitachi, Ltd., Tokyo, Japan) were used to observe the morphology of the nanoparticles. The size distribution, polydispersity index (PDI), and zeta potential were obtained using dynamic light scattering (DLS, Brookhaven Omni, Brookhaven Instruments Inc., Holtsville, NY, USA). The Fourier transform infrared spectrometer (FTIR, Nicolet iS10, Thermo Fisher Scientific Co., Ltd., MA, USA) was used to record the spectra of HA, BNPs@Ptx, and HA-BNPs@Ptx in the wavelength range of $500-4,000 \mathrm{~cm}^{-1}$. The drug loading (DL) and encapsulation efficiency (EE) of Ptx were quantified using a standard calibration curve measured at $229 \mathrm{~nm}$ on a visible ultraviolet spectrophotometer (L5S, INESA Analytical Instrument Co., Ltd., Shanghai, China, Supplementary Material, Methods 1.1 for details).

\section{Microwave-induced thermal effect and paclitaxel release of HA-BNPs@Ptx}

To evaluate the thermal effect of MW irradiation with sensitizer BML, 1 ml of HA-BNPs@Ptx or HA-NPs@Ptx suspensions was added to a 24-well plate and exposed to MW energy instrument (WZY-1, Beijing Muheyu Electronics Co., Ltd, Beijing, China). The suspensions were irradiated for $4 \mathrm{~min}$ at different MW power $\left(0.4,0.8,1.2\right.$, and $\left.1.6 \mathrm{~W} \mathrm{~cm} \mathrm{~cm}^{-2}, 450 \mathrm{MHz}\right)$. The temperature change in the HA-BNPs@Ptx suspension was recorded every minute using an infrared thermal mapping instrument (FLUKE 572-2, Hawk-IR International, Inc., Everett, WA, USA). Ptx release kinetics upon MW heating was evaluated by using visible ultraviolet spectrophotometer.

\section{In vitro biocompatibility and targeting ability of HA-BNPs@Ptx Cytotoxicity assay}

Mouse breast cancer cell line 4T1, mouse macrophage cell line J774, and human umbilical vein endothelial cells (HUVECs) were purchased from Cellcook (Guangzhou Cellcook Cell Biotechnology, Ltd., Guangzhou, Guangdong, China) and cultured according to the instructions of the supplier. 4T1 cells and HUVECs were seeded into a 96-well plate $\left(1 \times 10^{4}\right.$ cells per well $)$ and incubated overnight. Five duplicate holes were set in each group. Subsequently, $100 \mu \mathrm{l}$ of HA-BNPs@Ptx at Ptx concentrations $(2,5,10,20$, and $50 \mu \mathrm{g} / \mathrm{ml})$ was introduced to each group. The cytotoxicity of HA-BNPs@Ptx was examined by CCK-8 viability assay (Boster Biological Technology Co., Ltd., CA, USA). The optical density (OD) was measured at $450 \mathrm{~nm}$ by a Varioskan Flash microplate reader (Synergy Mx, BioTek Instruments, Inc., Winooski, VT, USA). The cytotoxicity treated with HA-NPs (without drug loaded NPs) was evaluated using the same method.

\section{Hemolytic activity}

Two percent suspension of chicken red blood cells was used to determine the hemolytic activity of HA-BNPs@Ptx. Phosphate buffer saline (PBS; $100 \mu \mathrm{l})$, double distilled water, or HA-BNPs@ Ptx suspensions at Ptx concentrations of 2, 5, 10, 20, and $50 \mu \mathrm{g} / \mathrm{ml}$ were added to erythrocyte suspensions $(100 \mu \mathrm{l})$. PBS was used as the negative control group, and double distilled water was used as the positive control group. All suspensions were incubated at $37^{\circ} \mathrm{C}$ for $1 \mathrm{~h}$ and centrifuged at $800 \times g$ for $10 \mathrm{~min}$. The OD was measured at $550 \mathrm{~nm}$ using the Varioskan Flash microplate reader.

\section{CD44 receptor mediated nanoparticle targeting}

4T1 cells were used to verify CD44 targeting ability of nanoparticles. The CD44 expression level of 4T1 cells was evaluated by an inverted fluorescence microscope (Supplementary Material, Methods 1.2 for details). The targeting test was performed using BNPs@Ptx, HA-BNPs@Ptx and HA-BNPs@Ptx with the cells in which CD44 receptor was pre-saturated by an excess amount of free HA (Zhang et al., 2020). To obtain stained nanoparticles, 1 ml of BNPs@Ptx or HABNPs@Ptx suspensions were stained with $10 \mu \mathrm{l}$ of DiO (1:100). After overnight culture, 4T1 cells were co-incubated with $100 \mu \mathrm{l}$ of BNPs@Ptx or HA-BNPs@Ptx for 6 h. The targeting ability was visualized by the inverted fluorescence microscope (AX10 imager A2/AX10 cam HRC, Carl Zeiss, Co., Ltd., Jena, Germany). Flow cytometry (FCM, Cyto Flex, Beckman Coulter, Inc., CA, USA) was used to further evaluate the ability of HA-BNPs@Ptx to target $4 \mathrm{~T} 1$ cells at different time intervals (30 min, 1, 3, 6, and $24 \mathrm{~h}$ ).

\section{In vitro cellular uptake and intracellular tracking}

To examine cellular uptake and intracellular trafficking, BNPs@ Ptx and HA-BNPs@Ptx were labeled with a red fluorescence probe Dil. Endocytosis/phagocytosis experiments were performed in 4T1 cells and J774 cells (murine macrophages), respectively. $4 \mathrm{~T} 1$ cells per well $\left(1 \times 10^{4}\right)$ were seeded in confocal dishes. After 24 h, $100 \mu$ l of DiI-BNPs@Ptx and DiI-HA-BNPs@ Ptx was added to co-incubate with $4 \mathrm{~T} 1$ cells for 1,3 , and $6 \mathrm{~h}$, respectively. At each time point, $20 \mu \mathrm{l}$ of Lyso Tracker Green was added to each confocal dish to stain endo/lysosomes. One and a half hours after incubation with Lyso Tracker Green, all cells were fixed with $4 \%$ paraformaldehyde for $10 \mathrm{~min}$. 4,6-Diamidino-2phenylindole (DAPI, blue, $10 \mu \mathrm{l}$ ) was finally added in the fixed cells to stain the nuclei. Confocal fluorescence images of the fixed cells were obtained by a laser scanning confocal microscope (LSCM, A1R + MP, Nikon Co., Tokyo, Japan).

J774 cells were treated in the same way as $4 \mathrm{~T} 1$ cells, except that $5 \times 10^{4}$ cells and $10 \mu \mathrm{l}$ of PMA $(100 \mathrm{ng} / \mathrm{ml})$ were added to each dish and incubated for $48 \mathrm{~h}$.

\section{In vitro synergistic anti-tumor ability}

Synergistic anti-cancer activity of thermal chemotherapy was evaluated with $4 \mathrm{~T} 1$ cells in vitro. The cells were seeded into a six-well plate $\left(7.5 \times 10^{5}\right.$ cells per well $)$ for $24 \mathrm{~h}$ and then incubated with 1) PBS, 2) HA-BNPs@Ptx, 3) MW, 4) BNPs@Ptx + MW, an5) HA-BNPs@Pd tx + MW for 6h. In order to optimize the thermal effects of nanoparticles, HA-BNPs@Ptx/4T1 cells were further exposed to MW irradiation at different power $(0.4,0.8$, 1.2 , and $1.6 \mathrm{~W} \mathrm{~cm}^{-2}$ ) for $1,2,3$, and $4 \mathrm{~min}$, respectively (see 
Supplementary Material, Methods 1.3 for details). After incubation, the cells of the last three groups were exposed to MW irradiation $\left(0.8 \mathrm{~W} \mathrm{~cm}^{-2}, 4 \mathrm{~min}\right)$. The anti-cancer activity of each group was qualitatively examined by using calcein-AM/ propidium iodide (PI) double stain kit (Beyotime Biotechnology ${ }^{\circledR}$ Inc., Suzhou, Jiangsu, China). After LIVE/DEAD staining of the cells, all cell samples were imaged under an inverted fluorescence microscope.

To quantitatively assess the synergistic chemo-thermal therapy, 4T1 cells were stained by Annexin V-(FITC)/PI apoptosis detection kit (4A Biotech Co., Ltd., Peking, China) and analyzed by flow cytometer.

\section{In vivo triple-negative breast cancer models, biodistribution, and targeting ability} $\mathrm{BALB} / \mathrm{c}$ mice (female, 18-20 g) were provided by Dashuo Biological Technology (Chengdu, Sichuan, China). All processes were in accordance with the Chinese Society of Laboratory Animals on animal welfare and approved by the Animal Use and Care Management Advisory Committee of West China Hospital of Sichuan University (Approval No. 2017014A). 4T1 cells $\left(1 \times 10^{6} /\right.$ wells $)$ were implanted into the second axillary mammary fat pad on the right side. When the volume of tumor reached about $100 \mathrm{~mm}^{3}$, 4T1 breast tumorbearing mice were randomly assigned into four groups (six mice per group): 1) DiI-BNPs@Ptx, 2) DiI-HA-BNPs@Ptx, 3) MW+DiI-BNPs@Ptx, and 4) MW +DiI-HA-BNPs@Ptx to compare the effect among non-targeted (EPR), CD44-targeted (active targeting), and MW irradiation (physical targeting). MW + DiI-BNPs@Ptx and MW +DiI-HA-BNPs@Ptx were treated with mild hyperthermia (4-min $\mathrm{MW}$ exposure at $0.8 \mathrm{~W} \mathrm{~cm}^{-2}$ ). Then nanoparticles $(0.5 \mu \mathrm{l} / \mathrm{g}$, each microliter of nanoparticle suspension contained $0.434 \mu \mathrm{g}$ of $\mathrm{Ptx}$ and $1.45 \mu \mathrm{g}$ of nanoparticles) were injected into $4 \mathrm{~T} 1$ breast tumor-bearing mice via tail vein. In vivo fluorescence images were collected before and after injection of nanoparticles at 1,3,6, and $24 \mathrm{~h}$ using the IVIS Spectrum system (Lumina XR, Caliper Life Sciences, Boston, MA, USA). To examine the biodistribution of nanoparticles, the main organs (heart, liver, spleen, lungs, and kidneys) and tumors were isolated from mice to perform ex vivo imaging using the same IVIS Spectrum.

Blood chemistry analysis of $\mathrm{BALB} / \mathrm{c}$ mice was made 7 days after intravenous injection with HA-BNPs@Ptx (Supplementary Material, Methods 1.4 for details).

\section{In vivo mild hyperthermia - the first microwave irradiation}

The first MW irradiation aimed to generate mild hyperthermia to alter the TME to increase the uptake of nanoparticles. Six mice in each group received mild hyperthermia at the tumor site with a MW power of $0.8 \mathrm{~W} \mathrm{~cm}$. The temperature of tumor was monitored in real time using an infrared thermal mapping instrument. After mild hyperthermia, DiI-HA-BNPs@Ptx $(0.5 \mu \mathrm{l} / \mathrm{g}$, each microliter of nanoparticle suspension contained $1.45 \mu \mathrm{g}$ of nanoparticles) was immediately injected into $4 \mathrm{~T} 1$ breast tumor-bearing mice via tail vein. The mice were euthanized, and the tumor tissue was removed after $24 \mathrm{~h}$. The retention of nanoparticles (red fluorescence) in tumor tissues was revealed using a pathological section scanner Pannoramic DESK (P-MIDI-P250, 3D HISTECH, Budapest, Hungary).

To further explore changes in TME, intratumoral perfusion and micro-vessel density of tumor were evaluated using contrastenhanced ultrasound imaging (CEUS) and CD31 immunohistochemical analysis. Microbubbles $(0.2 \mathrm{ml} / \mathrm{kg}$, SonoVueTM, Bracco, Italy) were injected via tail vein $24 \mathrm{~h}$ after the first MW irradiation. CEUS was performed using an ultrasound scanner (iU22, Koninklijke Philips N.V., Eindhoven, Netherlands) with a 12 - to $5-\mathrm{MHz}$ transducer. Subsequently, mice were euthanized, and tumors were sectioned and stained with CD31 (dilution 1:50, Wuhan Service Bio Co., Ltd., Wuhan, Hubei, China) to evaluate the micro-vessel density. The pathological sections were imaged using a fluorescence microscope.

\section{In vivo anti-tumor efficiency-the second microwave irradiation}

The second MW irradiation was dedicated to activate the sensitizer BML and to release the chemotherapy agent Ptx in the nanoparticles. Thirty 4T1 breast tumor-bearing mice were divided into five groups: PBS (control group, G1) (Tajbakhsh et al., 2018), first MW + HA-BNPs@Ptx (G2) (Thakur and Kutty, 2019), first $\mathrm{MW}+\mathrm{HA}-\mathrm{BNPs}+$ second MW (G3) (Mu et al., 2017), HA-BNPs@Ptx + second MW (conventional SDDS, G4) (Ding et al., 2021), and first MW + HA-BNPs@Ptx + second MW (G5) (Hu et al., 2020). After treatment with mild hyperthermia (4-min MW exposure at $0.8 \mathrm{~W} \mathrm{~cm}^{-2}, \mathrm{G} 2, \mathrm{G} 3$, and G5), PBS, HABNPs, or HA-BNPs@Ptx $(0.5 \mu \mathrm{l} / \mathrm{g}$, each microliter of nanoparticle suspension contained $0.434 \mu \mathrm{g}$ of $\mathrm{Ptx}$ ) was immediately injected into $4 \mathrm{~T} 1$ breast tumor-bearing mice intravenously. Twenty-four hours after injection, the mice received a second $\mathrm{MW}$ irradiation $\left(0.8 \mathrm{~W} \mathrm{~cm} \mathrm{~cm}^{-2}\right)$ for $4 \mathrm{~min}$. The tumor temperature was monitored in real time by infrared thermal mapping instrument. The body weight and tumor volumes were recorded every 3 days. The tumor suppression rate (TSR) was calculated using the following formula:

$$
\operatorname{TSR}(\%)=(\mathrm{Vc}-\mathrm{Vx}) / \mathrm{Vc} \times 100 \%
$$

where $\mathrm{Vc}$ is the volume of the PBS group, and Vx is the volume of the treatment group.

On day 18 after treatment, tumors and main organs were excised and fixed overnight in $10 \%$ buffered formalin. The tumors were sectioned and stained with H\&E, Ki-67 antibody, and TdT-mediated dUTP nick-end labeling (TUNEL) staining. The main organs were sectioned and stained with $\mathrm{H} \& \mathrm{E}$. The expression level of $\mathrm{CD}^{+}, \mathrm{CD}^{+}$, and $\mathrm{CD}^{+}$of immune cells in tumor tissues were evaluated by immunofluorescence. The slides of tumor tissues were incubated with Anti-CD3 Rabbit pAb (P22646,1:700), AntiCD4 Rabbit pAb (P06332 1:800), and Anti-CD8 Rabbit mAb (P10966, 1:500), following the standard procedure of Wuhan 
A

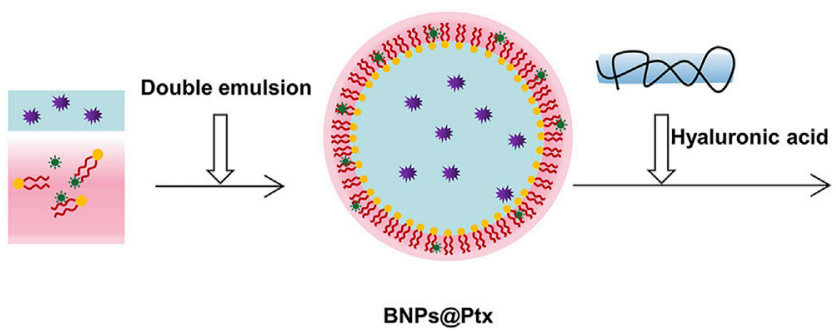

B

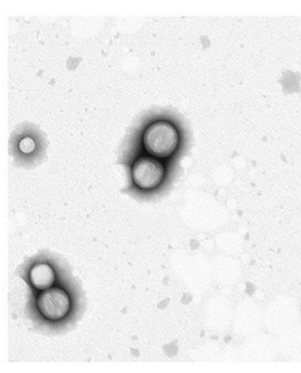

D

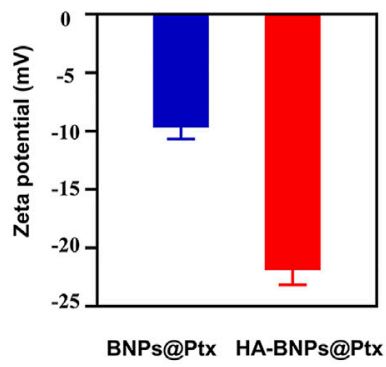

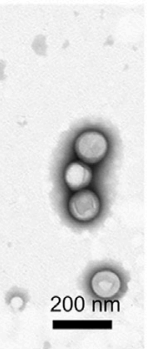

E

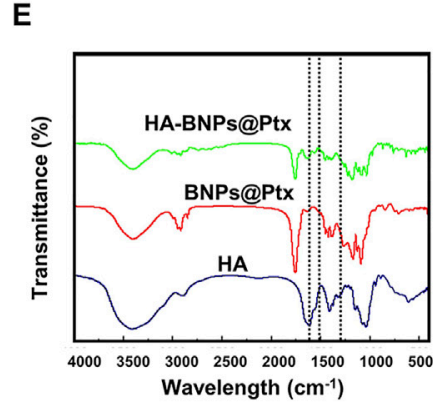

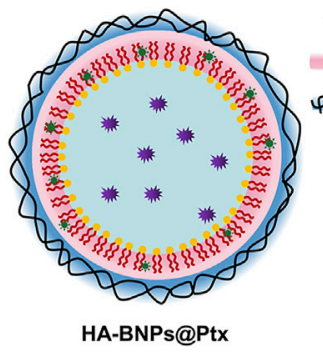

$\approx$ DPPC

$\mathrm{NH}_{2}$-PLGA-NH

$\oplus \infty D$ Hyaluronic Acid

* Ptx

BML

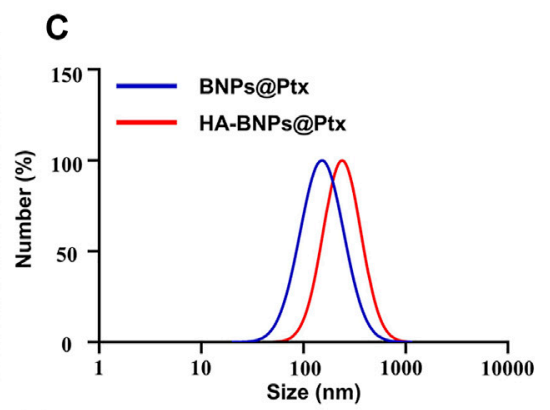

F

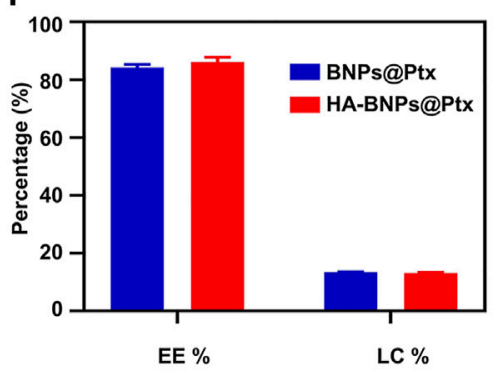

FIGURE 2 | Synthesis and characterization of nanoparticles used in synergistic cascade strategy study. (A) BNPs@Ptx preparation and hyaluronic acid (HA) conjugation. (B) Transmission electron microscopy (TEM) and scanning electron microscope (SEM) image of HA-BNPs@Ptx. (C) Size distribution and (D) zeta potentials of BNPs@Ptx and HA-BNPs@Ptx determined by dynamic light scattering (DLS) $(n=3)$. (E) Fourier transform infrared spectrometer (FTIR) spectrums of HA, BNPs@Ptx, and HA-BNPs@Ptx (amide bond: 1,656, 1,546, and 1,334 cm ${ }^{-1}$ ). (F) Encapsulation efficiency (EE) and loading capacity (LC) of Ptx in HA-BNPs@Ptx and BNPs@ Ptx determined by spectrophotometer $(n=3)$.

Service Bio Co., Ltd. (Wuhan, Hubei, China). The nucleus was labeled with DAPI. The images were obtained by using the pathological section scanner Pannoramic DESK.

In addition, peripheral blood was collected from treated mice before euthanasia to analyze the percentages of tumor antigen-specific $\mathrm{CD}^{+}, \mathrm{CD}^{+}$, and $\mathrm{CD}^{+}{ }^{+} \mathrm{T}$ cells. Serum cells were incubated with $3 \mu \mathrm{l}$ of FITC anti-mouse CD3 antibody, $5 \mu \mathrm{l}$ of $\mathrm{PE}$ anti-mouse CD4, and $10 \mu \mathrm{l}$ of APC anti-mouse CD8 (4A Biotech Co., Ltd., Peking, China) for $1 \mathrm{~h}$ at room temperature, respectively. Finally, the cells were resuspended in PBS and analyzed by flow cytometry and Flow Jo software.

\section{Statistical analysis}

All data were expressed as mean \pm standard deviation (SD). The statistical analysis was carried out with GraphPad Prism Version 8.0 software (GraphPad, USA). Comparisons among multiple groups were performed by one-way analysis of variance (ANOVA). Twogroup comparisons were performed by Student's $t$-test. Statistical significance was indicated by ${ }^{\star}$ for $p<0.05,{ }^{* *}$ for $p<0.01$, and ${ }^{\star *}$ for $p<0.001$.

\section{RESULTS}

\section{The characterization of HA-BNPs@Ptx}

Figure 2A illustrates the preparation procedure of nanoparticles HA-BNPs@Ptx. TEM and SEM images showed a core-shell structure of the nanoparticles with a mean diameter of about $150 \mathrm{~nm}$, spherical shaped, and with a smooth surface (Figure 2B). DLS revealed that HA-BNPs@Ptx was $203.30 \pm 7.51 \mathrm{~nm}$ and relatively homogenous (PDI: 0.172, Figure 2C).HA-BNPs@ Ptx was negatively charged with zeta potential of $-22 \mathrm{mV}$ (Figure 2D) and stable at neutral pH (Supplementary Figures S1A,B). The vibration absorption peaks of amide bonds at 1,656, 1,546 , and $1,334 \mathrm{~cm}^{-1}$ in FTIR spectrums confirmed the successful conjugation of HA to BNPs@Ptx nanoparticles (Figure 2E). Ptx loading capacity of HA-BNPs@Ptx 

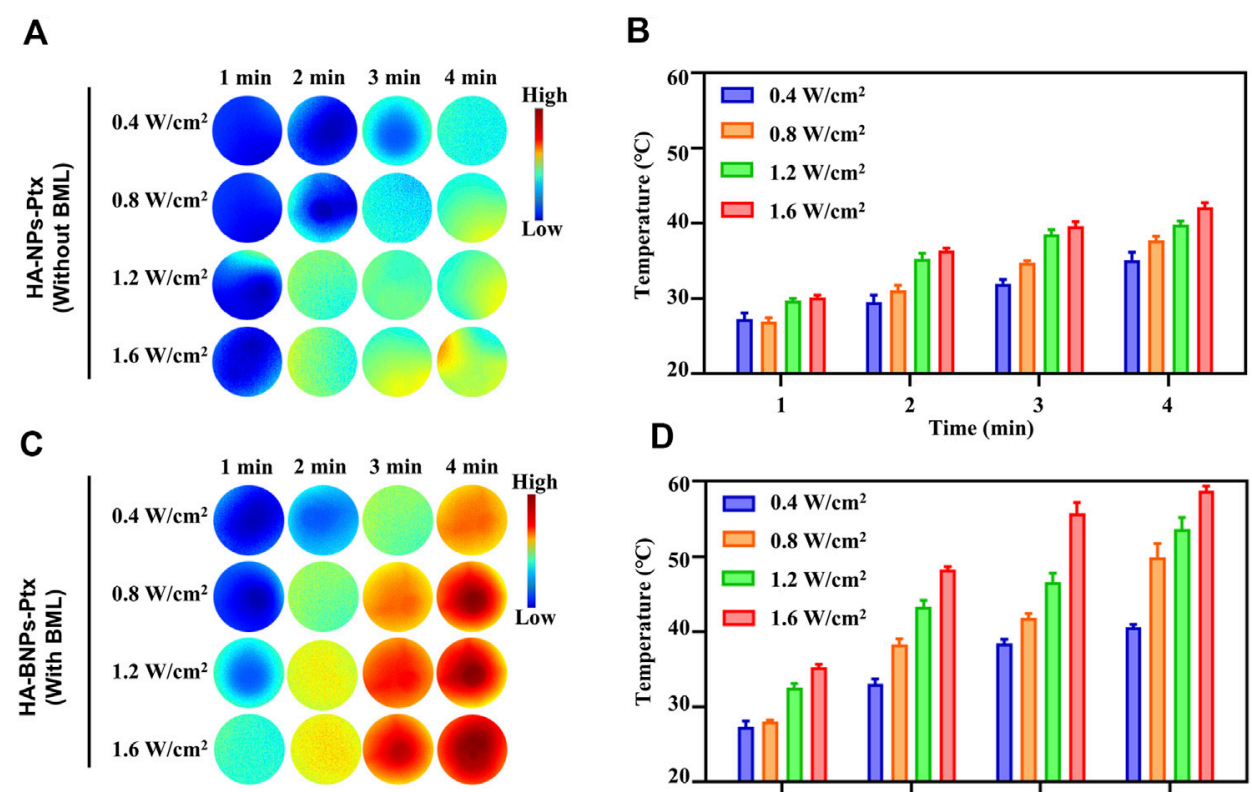

D

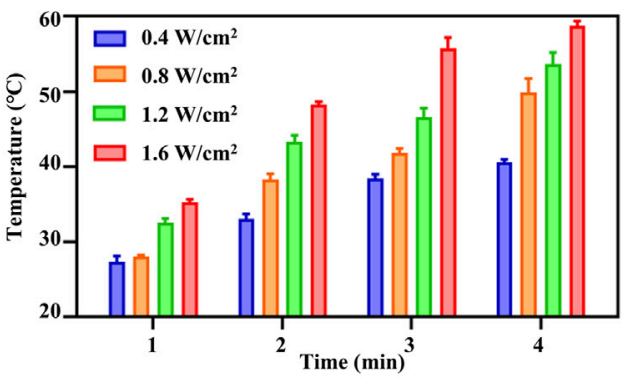

E

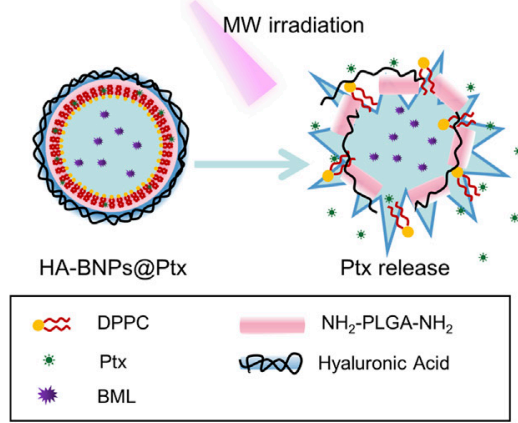

$\mathbf{F}$

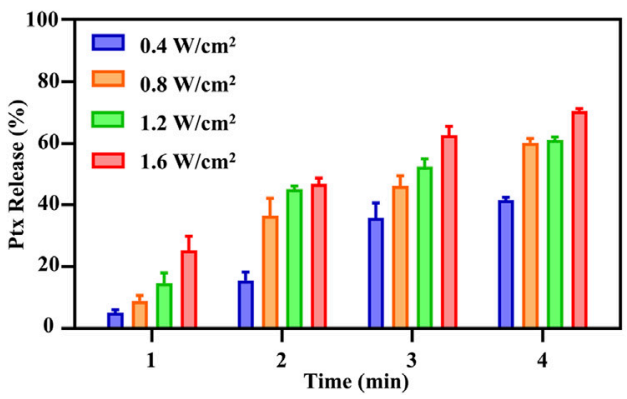

FIGURE 3 I In vitro evaluation of microwave (MW) thermal effect and paclitaxel (Ptx) release. (A, B) IR thermal imaging and temperature histogram of HA-NPs@Ptx [without thermosensitive agent 1-butyl-3-methylimidazolium-L-lactate (BML)] at different MW power and exposure time. (C, D) IR thermal imaging and temperature histogram of HA-BNPs@Ptx (with thermosensitive agent BML). (E) Schematic illustration of HA-BNPs@Ptx BML activation and Ptx release under MW irradiation. (F) In vitro release of Ptx from HA-BNPs@Ptx exposed at different MW power and irradiation time. The data are presented as mean \pm SD $(n=3)$.

determined by spectrophotometer was 13.17 wt.\%, corresponding to encapsulation efficiency of 85.62 wt.\% (Figure 2F).

\section{In vitro microwave-induced 1-butyl-3-methylimidazolium-L-lactate thermal effect and drug release}

In order to optimize the MW thermal effect and Ptx release, nanoparticle suspensions (HA-NPs@Ptx and HA-BNPs@Ptx) were exposed at different MW power and time. The infrared thermal imaging pictures showed that under the same irradiation, the heating effect of HA-NPs@Ptx without BML (Figures 3A,B) was significantly lower than that of HA-BNPs@Ptx with the sensitizer (Figures 3C,D). For HA-BNPs@Ptx, the temperature of the suspension increased rapidly from room temperature to $35.2^{\circ} \mathrm{C}\left(0.4 \mathrm{~W} \mathrm{~cm}^{-2}\right)$ and reached nearly $60^{\circ} \mathrm{C}\left(1.6 \mathrm{~W} \mathrm{~cm}^{-2}\right)$ under 4-min MW exposure. Due to the lack of BML, the temperature of HA-NPs@Ptx increased only to $30.4^{\circ} \mathrm{C}$ at $0.4 \mathrm{~W} \mathrm{~cm}^{-2}$ and $41.8^{\circ} \mathrm{C}$

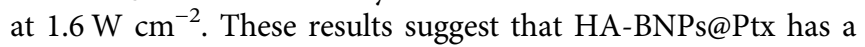
greater potential to trigger Ptx release in combination with thermal chemotherapy.

Figures 3E,F show the release of Ptx from HA-BNPs@Ptx upon MW irradiation. The Ptx solution standard curve was established according to previous reports to quantify Ptx (Supplementary Figure S2) (Furman et al., 2017). A maximum release of $70 \%$ was obtained at $1.6 \mathrm{~W} \mathrm{~cm} \mathrm{~cm}^{-2}$ for $4 \mathrm{~min}$. At this condition, the temperature was very high $\left(58^{\circ} \mathrm{C}\right.$, Figure 3D), and this would directly induce tumor necrosis (Seynhaeve et al., 2020; Pan et al., 2021). In order to avoid excessive heat and demonstrate our synergistic cascade strategy, a lower $\mathrm{MW}$ power of $0.8 \mathrm{~W} \mathrm{~cm}^{-2}$ was chosen for subsequent in vitro and in vivo studies. 

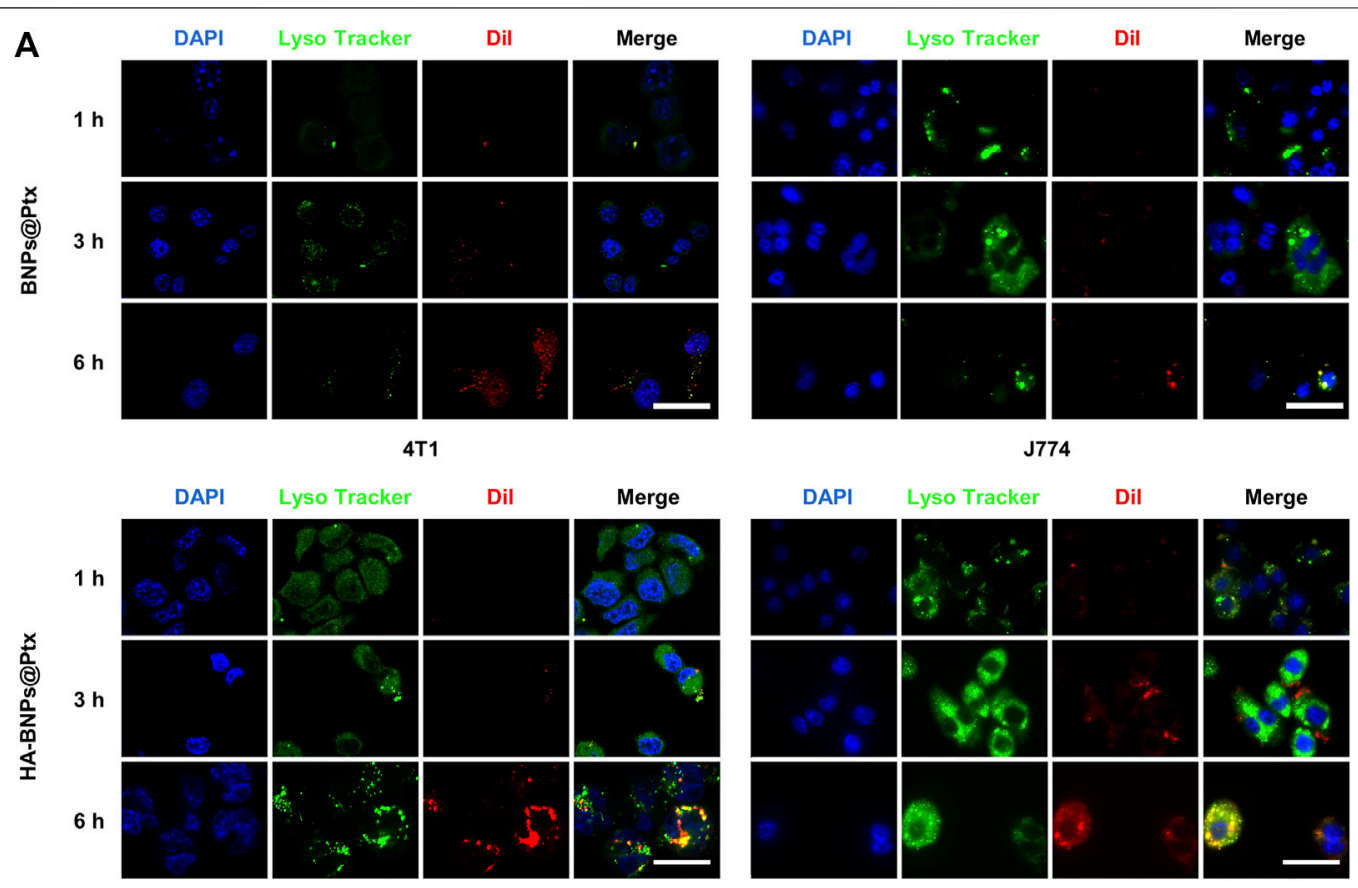

DAPI Lyso Tracker
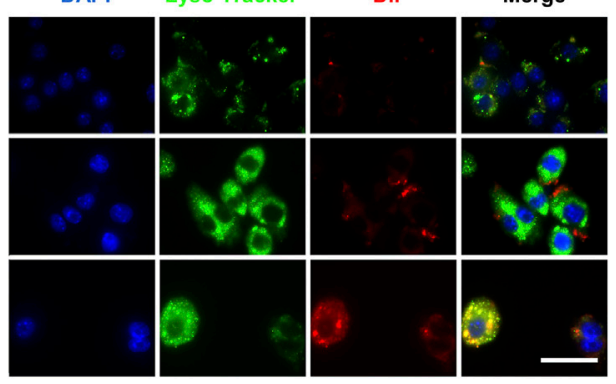

B

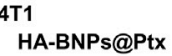

MW
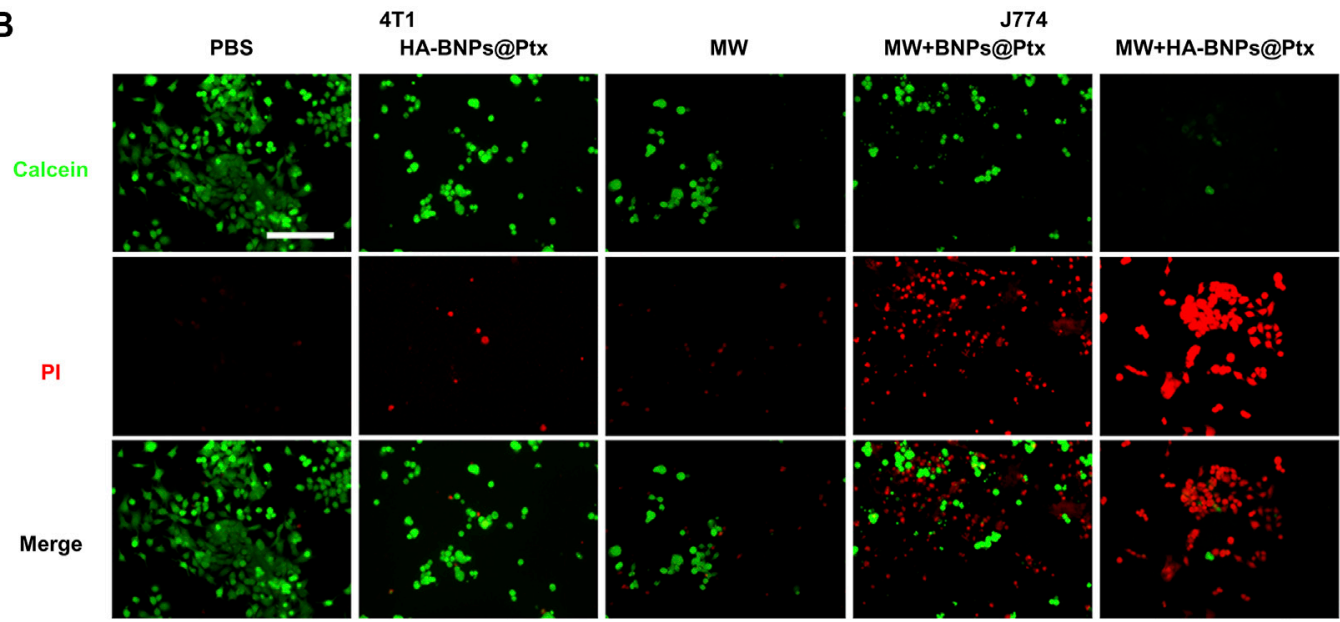

C

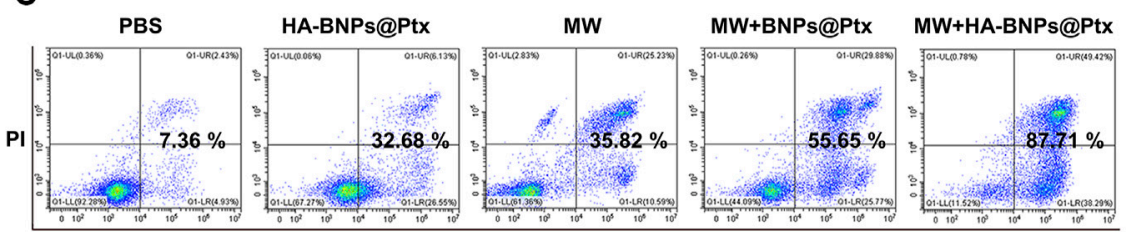

D

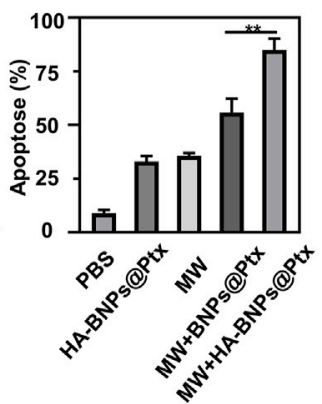

FIGURE 4 | In vitro endocytosis and apoptosis studies. (A) Representative confocal imaging of 4T1 breast cancer cells and J774 macrophages incubated with BNPs@Ptx and HA-BNPs@Ptx for 1, 3, and 6 h at 37 C. The cell nuclei were stained by 4,6-diamidino-2-phenylindole (DAPI) (blue), the endo/lysosomes by Lyso Tracker Green (green), and HA-BNPs@Ptx were labeled with Dil (red). Scale bars represent $20 \mu \mathrm{m}$. (B) Fluorescence images of 4 T1 cells treated with phosphate buffer saline (PBS), HA-BNPs@Ptx, MW, MW + BNPs@Ptx, or MW + HA-BNPs@Ptx. Live cells were stained by Calcein-AM (green) and dead cells by PI (red). Scale bars represent $50 \mu \mathrm{m}$. (C) After treatment, early and late apoptotic cells were determined by flow cytometry using the Annexin $V$-Alexa Fluor 488/Propidium lodide Apoptosis Detection kit. (D) Total cell apoptosis rate was detected by flow cytometry. The data are presented as mean \pm SD $\left(n=3\right.$, $\left.{ }^{* *} p<0.01\right)$. 


\section{In vitro targeting ability, cellular uptake, and anti-tumor effect}

Hyaluronic acid, as a CD44-specific ligand, is widely used in various targeted drug delivery systems for TNBC (Zhang et al., 2020; Sun et al., 2019). The expression of CD44 on 4T1 cells was verified (Supplementary Figure S3A), and the specific targeting ability of nanoparticles to $4 \mathrm{~T} 1$ cells was compared between HABNPs@Ptx and BNPs@Ptx (Supplementary Figure S3B). Supplementary Figure S3A reveals that the nanoparticles contain HA bonded fairly to $4 \mathrm{~T} 1$ cells, and the binding was specific because the binding could be inhibited by adding the free hyaluronic acid to $4 \mathrm{~T} 1$ cells before the incubation with the nanoparticles (Cerqueira et al., 2017). Non-specific binding could be observed with BNPs@Ptx, but the number of fluorescent nanoparticles was significantly less than that of HA-BNPs@Ptx, suggesting that the specific binding of HA and CD44 could be used to improve the cellular internalization efficiency of nanoparticles (Supplementary Figure S3B).

The cellular uptake of HA-BNPs@Ptx was evaluated with 4T1 cells and J774 cells. The cell nuclei were stained by DAPI (blue), the endo/lysosomes were stained by LysoTracker Green (green), and the nanoparticles were labeled with DiI (red). Figure 4A shows that BNPs@Ptx and HA-BNPs@Ptx were taken up both by 4T1 and J774 cells, and stayed in endo/lysosomes. However, HABNPs@Ptx leads to higher fluorescent intensity compared with BNPs@Ptx. Furthermore, the uptake of HA-BNPs@Ptx by 4T1 cells was quantified using flow cytometry. A maximum uptake was observed after $6 \mathrm{~h}$ of incubation (Supplementary Figure S4). A lower fluorescent intensity obtained at $24 \mathrm{~h}$ suggests probably a partial degradation of nanoparticles in endo/lysosomes.

The synergistic antitumor effect of MW thermal chemotherapy was studied qualitatively by confocal microscope and quantitatively by flow cytometry. In vitro hyperthermia experiments indicate that MW irradiation alone was sufficient to induce apoptosis of $4 \mathrm{~T} 1$ tumor cells (Supplementary Figure S5), and the effect increased with increasing exposure power and time. Figure $\mathbf{4 B}$ shows that the combination of MW and HA-BNPs@Ptx resulted in the most significant cell damage compared with the other tested conditions (PBS，BNPs@Ptx，MW，MW + BNPs@Ptx). The 4T1 cell apoptosis analyzed by flow cytometry presented similar results (Figures 4C,D). The apoptosis was less than $8 \%$ for the control group (PBS), 32\% for HA-BNPs@Ptx alone, 35\% for MW irradiation only, $55.6 \%$ for MW + BNPs@Ptx, and $87 \%$ for MW + HA-BNPs@Ptx. These results suggest that there was a synergistic effect by combining hyperthermia and Ptx-loaded nanoparticles, and also confirm the superiority of CD44targeted nanoparticles compared with non-targeting nanoparticles.

The biocompatibility of HA-BNPs@Ptx and HA-NPs was investigated by cell counting kit8 (CCK8) assay with HUVEC and $4 \mathrm{~T} 1$ breast cancer cells. A high cell viability (>92\%) was obtained after incubation with HA-BNPs@Ptx and HA-NPs (without Ptx and BML) for $24 \mathrm{~h}$ for the two cell lines (Supplementary Material, Supplementary Figure S6). Supplementary Figure S7 shows that the hemolysis rate induced by HA-BNPs@Ptx is less than 5\%. This confirmed that the nanoparticles had good biocompatibility and also showed good stability, with no leakage of Ptx during 24-h incubation (Nave et al., 2016).

\section{In vivo targeting ability and biodistribution of HA-BNPs@Ptx compared with BNPs@Ptx (without the absence and presence of first microwave)}

To verify the targeting ability and biodistribution of HA-BNPs@ Ptx, 4T1 breast tumor-bearing mice model was established in the second axillary mammary fat pad on the right side. HA-BNPs@ Ptx and BNPs@Ptx were injected intravenously into 4T1 breast tumor-bearing mice, respectively. The targeting ability and biodistribution of nanoparticles were studied in real-time using a whole animal fluorescence imaging. The intensity of HA-BNPs@Ptx and BNPs@Ptx at tumor sites reached the maximum level at $24 \mathrm{~h}$ (Figure 5A). Compared with the nontargeted BNPs@Ptx group, the HA-BNPs@Ptx group exhibited significantly higher uptake in the tumor, almost two times higher (Figure 5B) due to the presence of HA active targeting. For untargeted BNPs@Ptx, the first MW exposure also improved significantly the EPR effect, the tumor uptake comparable with HA-BNPs@Ptx without the first MW exposure. The first MW + HA-BNPs@Ptx had the highest tumor retention rate, which is 1.7 times that of the first MW + BNPs@Ptx and HABNPs@Ptx, and almost four times that of BNPs@Ptx alone (EPR effect) both at 6 and $24 \mathrm{~h}$ after the injection of nanoparticles. Tumor tissues and major organs (heart, lung, kidney, liver, and spleen) were harvested for ex vivo imaging (Figure 5C). The fluorescence intensity of tumor tissues was also two times higher for the targeted nanoparticles than for non-targeted nanoparticles (Figure 5D). For other organs, comparable results were observed in the liver and spleen; a stronger fluorescence was observed in the lungs for HA-BNPs@Ptx, probably suggesting a higher CD44 expression in the lungs of the breast cancer model.

\section{In vivo mild hyperthermia (first microwave)-mediated tumor uptake of HA-BNPs@Ptx}

The purpose of the first MW irradiation was to pretreat animals to modify TME, so as to improve the tumor uptake of nanoparticles (Yang and Gao, 2017; Hynynen, 2018). In order to understand the mechanism of the pretreatment of MW prior to the injection of HA-BNPs@Ptx, the animal was continuously monitored by infrared thermography to control the temperature change in tumors. Figures $\mathbf{6 A}, \mathbf{B}$ show that the temperature of tumors was $36.7 \pm 0.2^{\circ} \mathrm{C}$ before the first MW irradiation and increased to $43.8 \pm 0.4^{\circ} \mathrm{C}$ after $4 \mathrm{~min}$ of $\mathrm{MW}$ irradiation $(0.8 \mathrm{~W}$ $\mathrm{cm}^{-2}$ ). After the treatment with MW, the nanoparticles DiI-HABNPs@Ptx were immediately injected into mice through the tail vein $(\sim 0.5 \mu \mathrm{l} / \mathrm{g})$. Twenty-four hours after treatment, the hyperthermia effect was measured by one of the following three methods. First, red fluorescence-labeled nanoparticles 
A
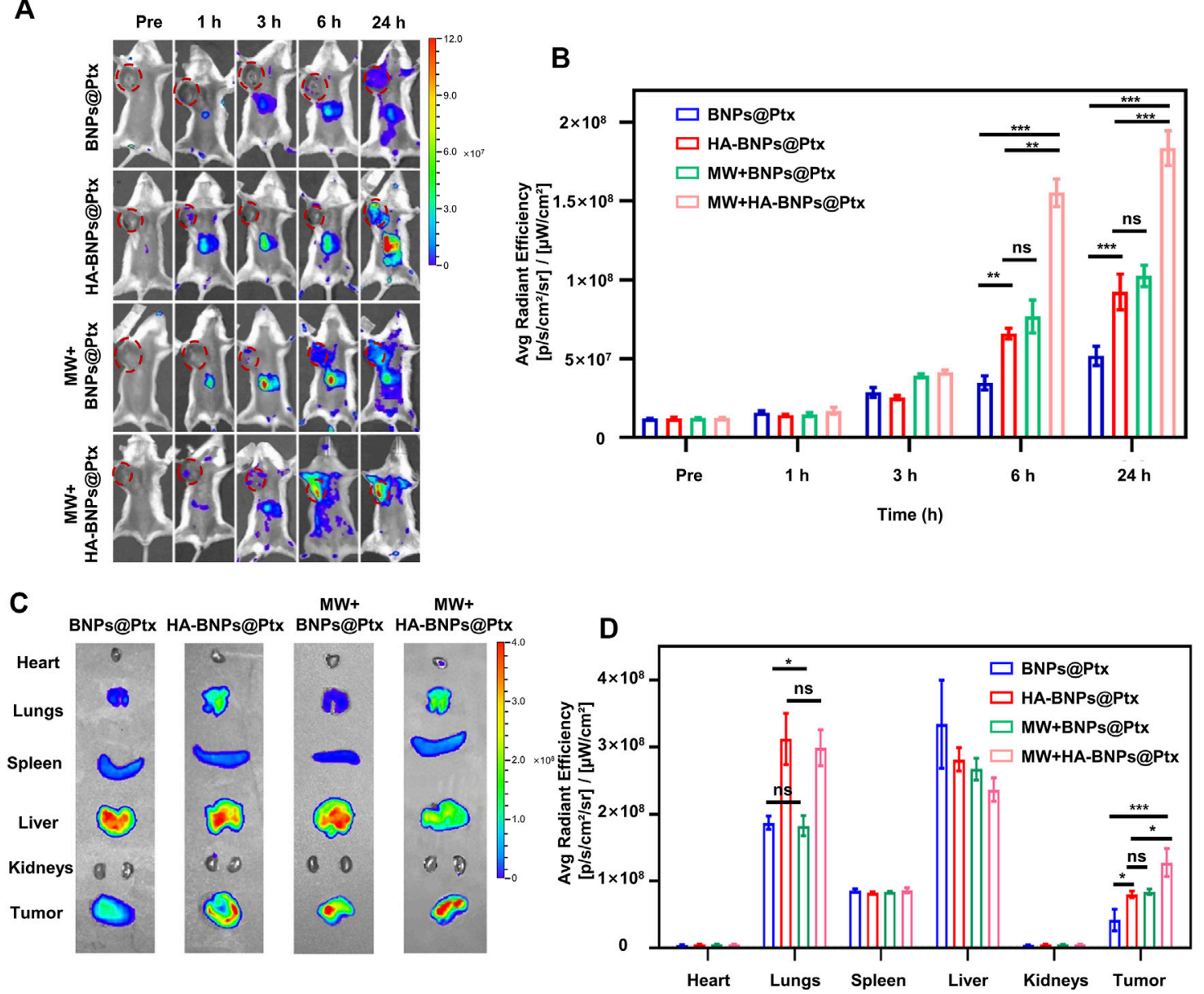

FIGURE 5 | In vivo targeting ability and biodistribution of nanoparticles. (A) Representative in vivo fluorescence images of $4 \mathrm{~T} 1$ breast tumor-bearing mice obtained at pre-injection, 1, 3, 6, and $24 \mathrm{~h}$ after intravenous injection of Dil-BNPs@Ptx and Dil-HA-BNPs@Ptx $(0.5 \mu \mathrm{l} / \mathrm{g})$ with or without first MW irradiation. (B) Average radiant intensity determined from in vivo fluorescence imaging for tumor-targeted (Dil-HA-BNPs@Ptx) and non-targeted (Dil-BNPs@Ptx) nanoparticles with or without first MW irradiation. (C) Ex vivo fluorescence images of main organs and tumor collected from tumor-bearing mice $24 \mathrm{~h}$ after injection of nanoparticles. (D) Average radiant intensity of main organs and tumor determined from ex vivo fluorescence imaging for four animal groups at $24 \mathrm{~h}$ after injection of nanoparticles. The data are presented as mean $\pm \mathrm{SD}\left(n=6\right.$, ns, not significant; $\left.{ }^{*} p<0.05,{ }^{* *} p<0.01,{ }^{\star \star *} p<0.001\right)$.

that accumulated at the tumor site were detected by tissue sections (Figures 6C,D). Second, contrast-enhanced ultrasound (CEUS) imaging was performed to measure the change in intratumoral blood flow (Figures 6E,F). Third, the morphology of tumor vasculature was examined using CD31 immunofluorescent staining (Figures 6G,H).

The results showed that the retention rate of nanoparticles in the tumors irradiated by MW increased by seven times compared with the tumors not irradiated (Figures 6C,D). The echo intensity of CEUS showed that the pretreatment of tumor and its surrounding tissue by MW irradiation improved significantly intratumoral perfusion (Figures 6E,F). CD31 immunofluorescence images also showed increased tumor vascular density and/or intratumoral vasodilatation inside the tumor compared with that of tumors not irradiated (Figure 6F). Figure 6G showed that $24 \mathrm{~h}$ after MW exposure, the percentage of effective tumor vessels increased by a factor of about fourfold, from $3.8 \pm 1.5 \%$ to $15.3 \pm 4.3 \%$. These results suggest that the pretreatment of tumors by mild hyperthermia can improve the accessibility of nanoparticles to tumors, possibly by reducing interstitial fluid pressure, dilating and increasing tumor blood vessels, and thus improving intratumoral perfusion (Chen et al., 2019).

\section{In vivo evaluation of synergistic cascade antitumor effect}

The synergistic cascade antitumor effect was achieved by combining two-step MW irradiations with target nanoparticles HA-BNPs@Ptx (Figure 7A). The tested conditions included group 1 (G1), PBS; group 2 (G2), first MW + HA-BNPs-Ptx; group 3 (G3), first $\mathrm{MW}+\mathrm{HA}$ BNPs + second MW; group 4 (G4), HA-BNPs-Ptx + second MW, and group 5 (G5) with first MW + HA-BNPs-Ptx + second 

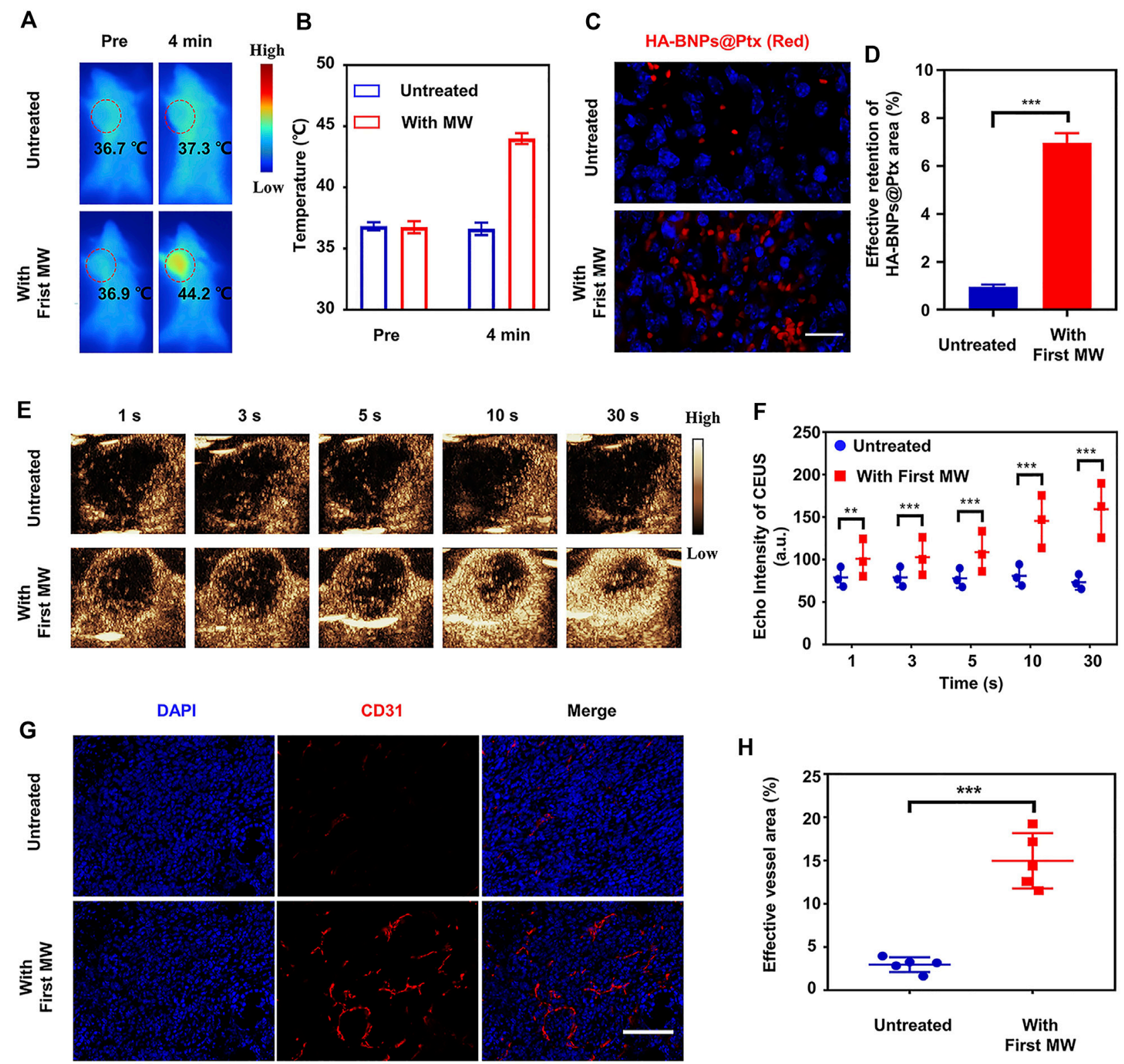

H

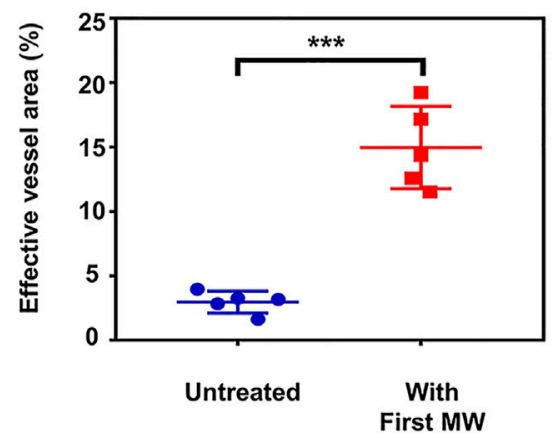

FIGURE 6 | In vivo mild hyperthermia effects on nanoparticle uptake and retention, intratumoral flow, and tumor vasculature on 4T1 breast tumor-bearing mice. (A) Representative IR thermal imaging of mice with or without MW irradiation $\left(0.8 \mathrm{~W} \mathrm{~cm}^{-2}, 4 \mathrm{~min}\right)$. (B) Average tumor temperature before and after MW irradiation ( $\left.n=3\right)$. (C) Representative fluorescence images of uptake/retention of Dil-HA-BNPs@Ptx in tumor-bearing mice untreated and treated with MW irradiation. Images were obtained at $24 \mathrm{~h}$ after the treatment. (D) Dil-HA-BNPs@Ptx quantitative analysis of excised tumors collected from mice $24 \mathrm{~h}$ after treatment $(n=3)$. (E) $/ n$ vivo contrastenhanced ultrasound images obtained at different time-points after injection of microbubble contrast agent SonoVue ${ }^{\mathrm{TM}}$. (F) Increased intratumoral perfusion was confirmed by quantifying contrast-enhanced echo intensity of MW-treated and untreated 4T1 tumors $(n=3)$. (G) Immunofluorescence expression of CD31 showed vascular dilation or opening within tumors, which was consistent with CEUS imaging finding. (H) Compared with untreated 4T1 tumor, the area of tumor blood vessels increased four times after MW irradiation. Scale bars represent $50 \mu \mathrm{m}$. The data are presented as mean $\pm \operatorname{SD}\left(n=5,{ }^{* *} p<0.01,{ }^{* \star *} p<0.001\right)$.

MW. Notice the difference between G2 and G5; G2 did not receive a second MW irradiation (nanoparticles); the difference between G3 and G5 is that the former contained no Ptx (thermal therapy); the difference between G4 and G5 is that G4 did not receive the first MW irradiation (tumor pre-treatment). The group (G3, G4, and G5) that received a second MW was irradiated under the same conditions as the first one ( $0.8 \mathrm{~W} \mathrm{~cm}^{-2}$ for $\left.4 \mathrm{~min}\right)$, and the tumor temperature was monitored in real time by an infrared thermal mapping instrument. The exposed temperature of G3 and G5 under second MW irradiation were all up to $51^{\circ} \mathrm{C}$ (Supplementary Material, Figures 7B,C). G4 reached to approximately $45^{\circ} \mathrm{C}$. This indicates that BML encapsulated in HA-BNPs-Ptx provided supplement heating.

Figure 7D shows that throughout the treatment period, there was no significant change in animal body weight in all groups, 

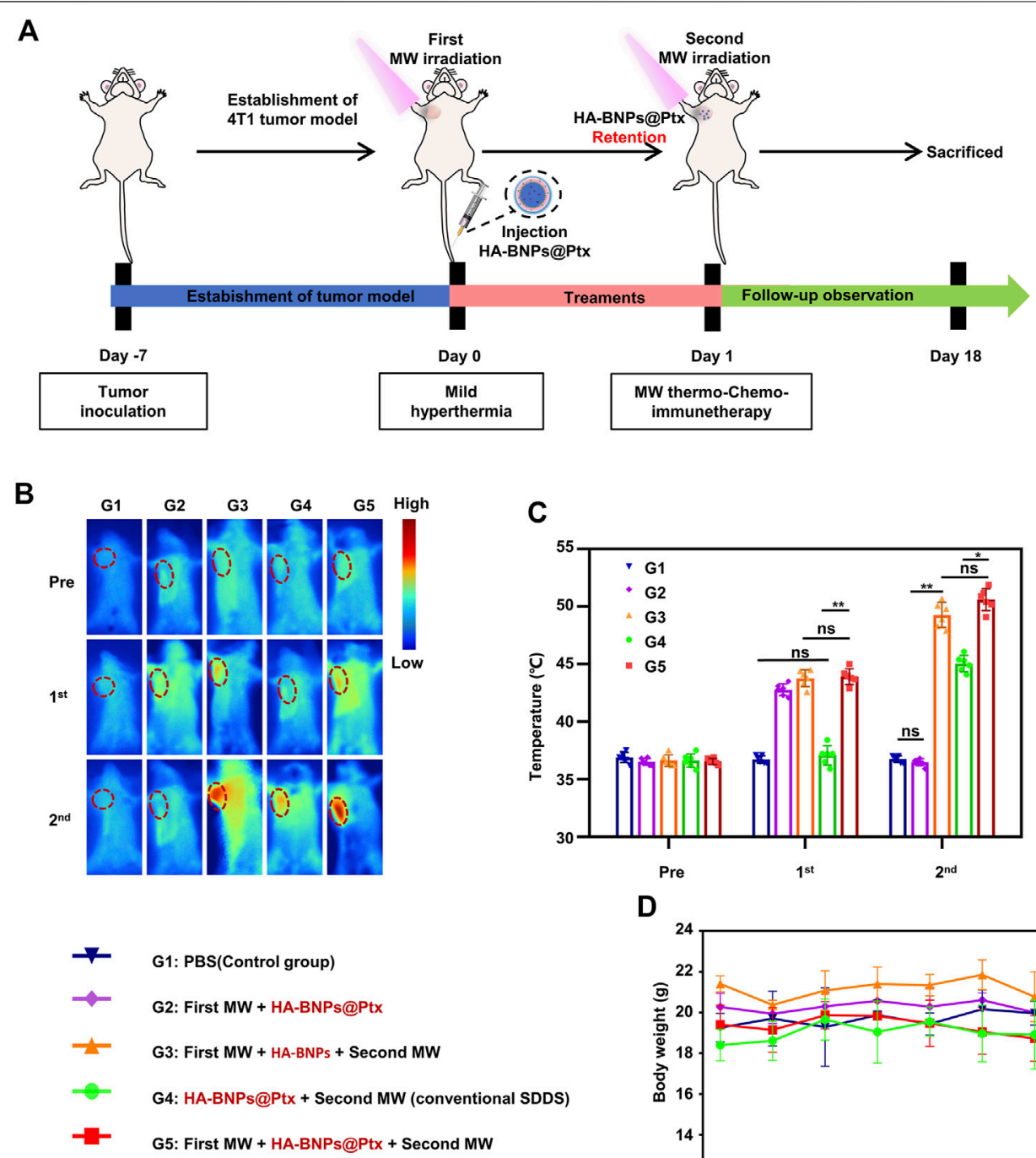

D

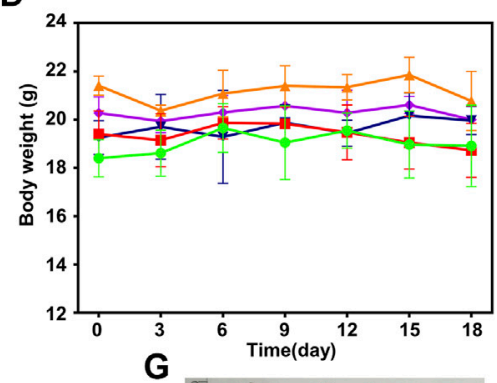

E
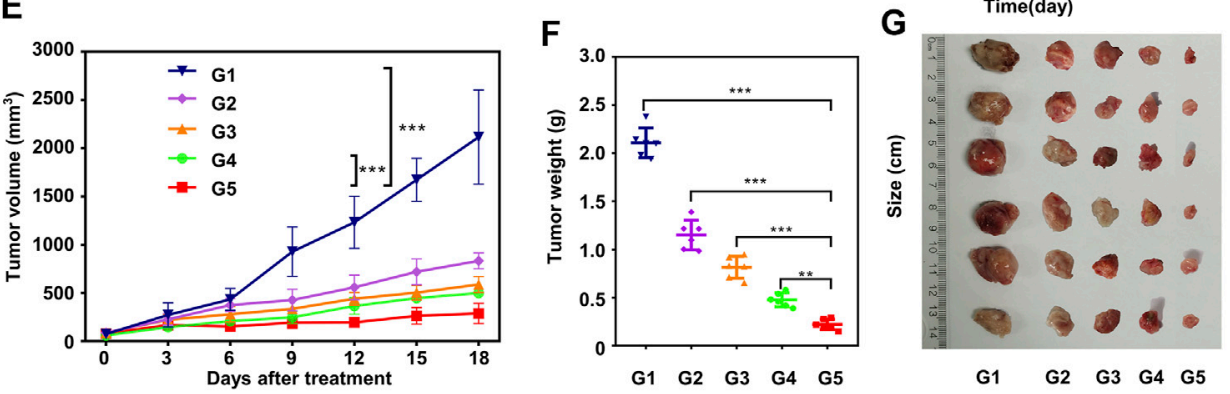

FIGURE 7 | In vivo study of the antitumor effect of synergistic cascade strategy (double hyperthermia + nanomedicine). (A) Schematic illustration of the treatment process of $4 \mathrm{~T} 1$ breast tumor-bearing mice by synergistic cascade strategy. (B) Representative IR thermal images of $4 \mathrm{~T} 1$ tumor-bearing mice treated with different conditions. MW irradiation was performed at $0.8 \mathrm{~W} \mathrm{~cm}^{-2}$ for $4 \mathrm{~min}$. (C) Changes in tumor temperature before and after first and second microwave exposure for different treatment mice groups (G1-G5). (D, E) Body weight tumor growth of mice after SCS treatment, evaluated every 3 days. (F, G) Changes in tumor volume and representative photograph of excised tumor of the $4 \mathrm{~T} 1$ breast tumor-bearing mice 18 days after the treatment (G1-G5). The data are presented as mean $\pm \mathrm{SD}(n=6$, ns, not significant; ${ }^{* *} p<0.01,{ }^{* \star *} p<0.001$ ).

indicating that all treatment groups were well tolerated. Compared with the other groups, the tumor volume of the G1 group increased rapidly, up to about $2,000 \mathrm{~mm}^{3}$ (Figures 7E-G).
The antitumor effect of the G5 group was the best, with the inhibition rate of tumor growth up to $88 \%$. G2 and G3 inhibited tumor growth by $58.2 \%$ and $67.5 \%$, respectively. G4 also has a 


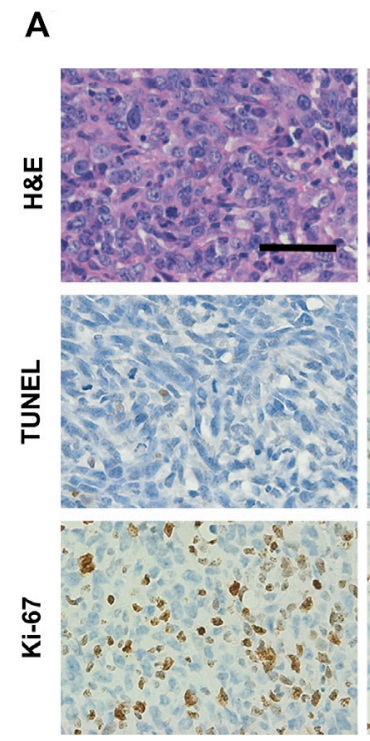

G1

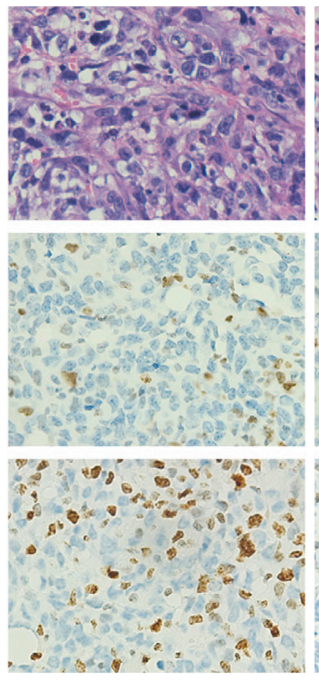

G2
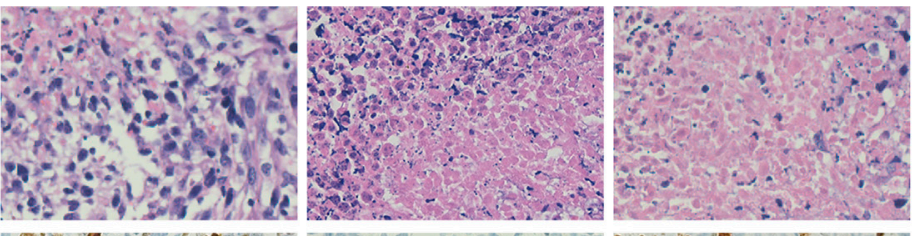

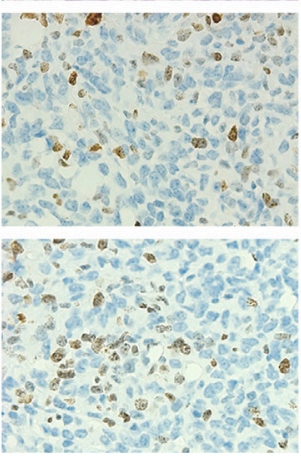

G3

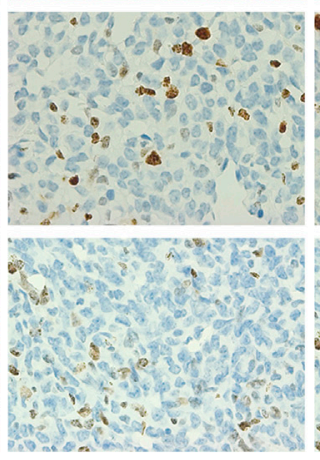

G4

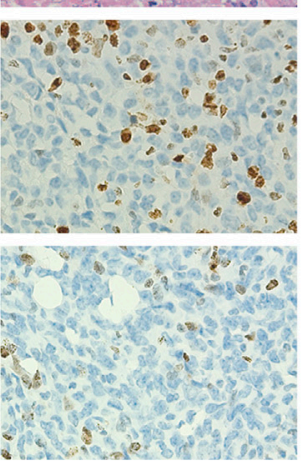

G5
B

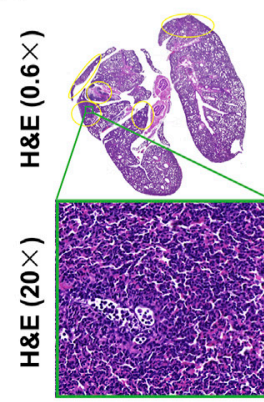

G1

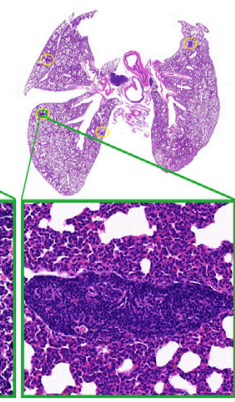

G2

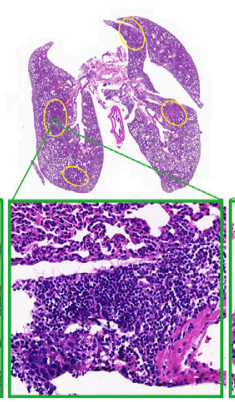

G3

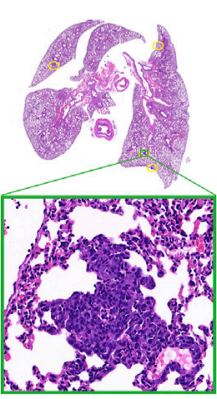

G4

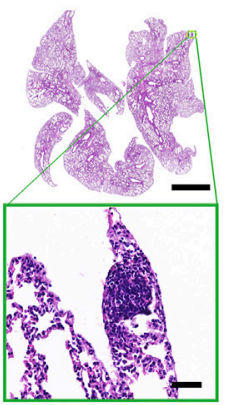

G5

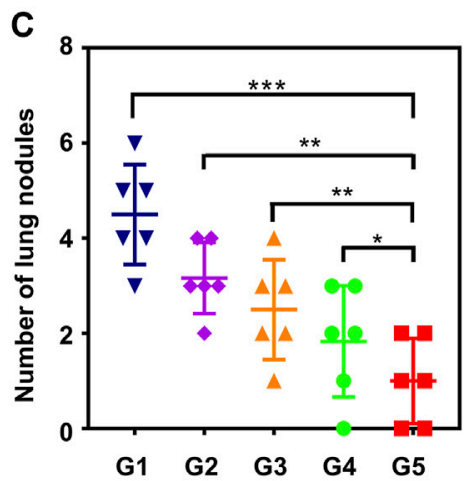

FIGURE 8 | In vivo study of the antitumor and anti-metastasis effect of synergistic cascade strategy. (A) Hematoxylin and eosin (H\&E) staining, Ki-67 staining, and TUNEL staining images of tumor tissue after different treatments (G1-G5) for 18 days. Scale bars represent $50 \mu \mathrm{m}$. (B) H\&E staining of lung metastatic sites (yellow borders) of $4 \mathrm{~T} 1$ tumors. Scale bars represent $2,000 \mu \mathrm{m}$ for $0.6 \times$ and $50 \mu \mathrm{m}$ for $20 x$, respectively. (C) The number of pulmonary metastatic nodules after various treatments (G1-G5) was calculated according to H\&E staining results. The data are presented as mean $\pm \mathrm{SD}\left(n=6,{ }^{\star \star} p<0.01,{ }^{\star \star \star} p<0.001\right)$.

good antitumor effect; the inhibition rate of tumor growth was about $72.5 \%$.

The degree of tumor tissue damage was evaluated by immunostaining using $\mathrm{H} \& \mathrm{E}$, proliferation marker Ki-67. and TUNEL essays. The results showed that the most severe morphological change and necrosis from tumor slices were observed in G5 (Figure 8A top). G5 significantly increased the apoptosis of cancer cells (Figure 8A middle) and had the lowest stained cells by Ki-67 (Figure 8A bottom). The pathological results were consistent with the tumor size and volume, indicating that G5 had the best antitumor effect.

Metastasis is a major challenge for TNBC treatment (Li et al., 2020). 4T1 breast tumor-bearing mice treated with a combination of two-step MW irradiation with HA-BNPs@Ptx exhibited a reduction in spontaneous lung metastasis (Figures 8B,C). Intratumoral immune profiling was performed in the five groups through immunofluorescence staining (Figures 9A-D).
The proportions of $\mathrm{T}$ cells $\left(\mathrm{CD}^{+}\right)$were increased in the treated group compared with G1. Among T cells, the percentages of $\mathrm{CD}^{+} \mathrm{T}$ cells and $\mathrm{CD} 8^{+} \mathrm{T}$ cells were significantly increased in the G5. Analysis of peripheral blood by flow cytometry was consistent with the above results (Figures 9E,F). These results suggest that sequential treatment of SCS can stimulate and recruit $\mathrm{CD} 4^{+} \mathrm{CD} 8^{+}$ $\mathrm{T}$ cells in breast cancer tissues and, thus, effectively inhibit spontaneous lung metastasis.

Subsequently, cytotoxicity of HA-BNPs-Ptx was investigated in vivo. There was no difference in liver and kidney function (analysis of serum levels for ALT, AST, BUN, and CRE) between the PBS group and the HA-BNPs-Ptx group (Supplementary Figure S8). This indicates that the nanoparticles have good biocompatibility. The histological analysis staining of main organs tissue was done by hematoxylin and eosin (H\&E) (Supplementary Figure S9). After the treatment to study the damage in mice, no tissue necrosis was observed in the main 
A

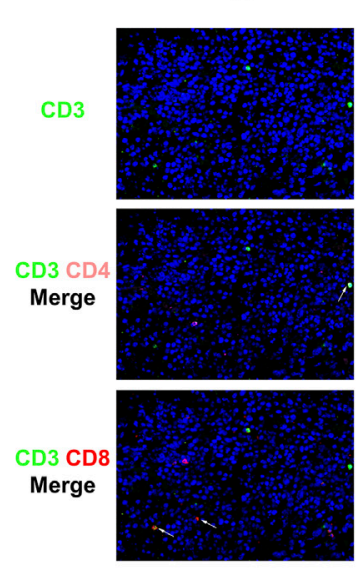

B

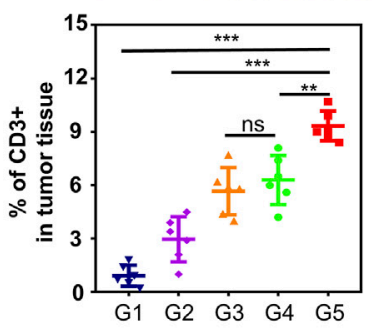

G2
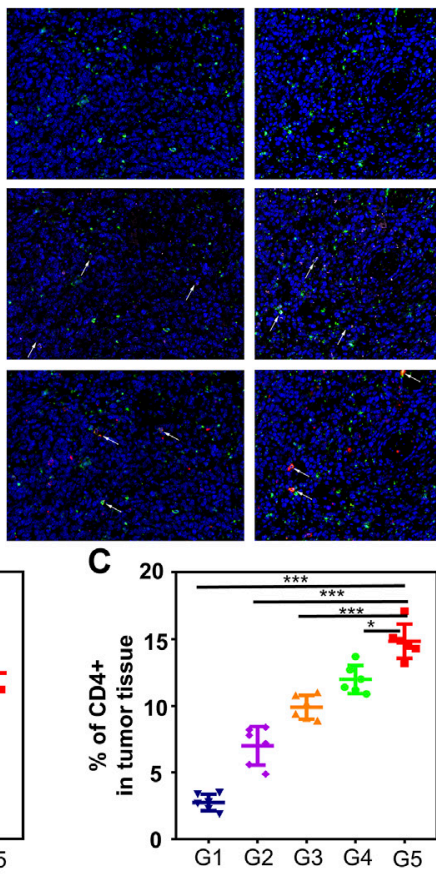

G3
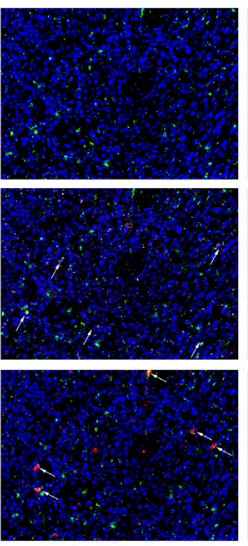

D
G4
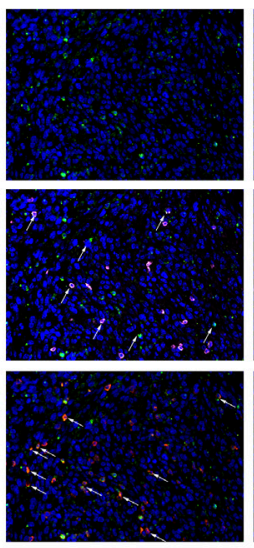

D

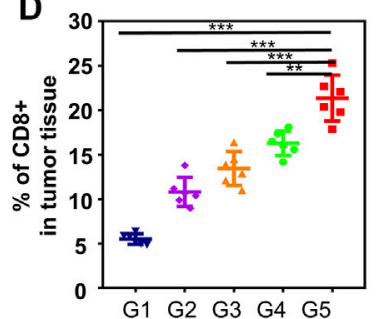

G1 G2 G3 G4 G5
G5
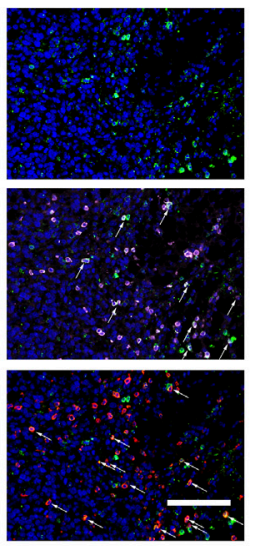

$\mathbf{F}$
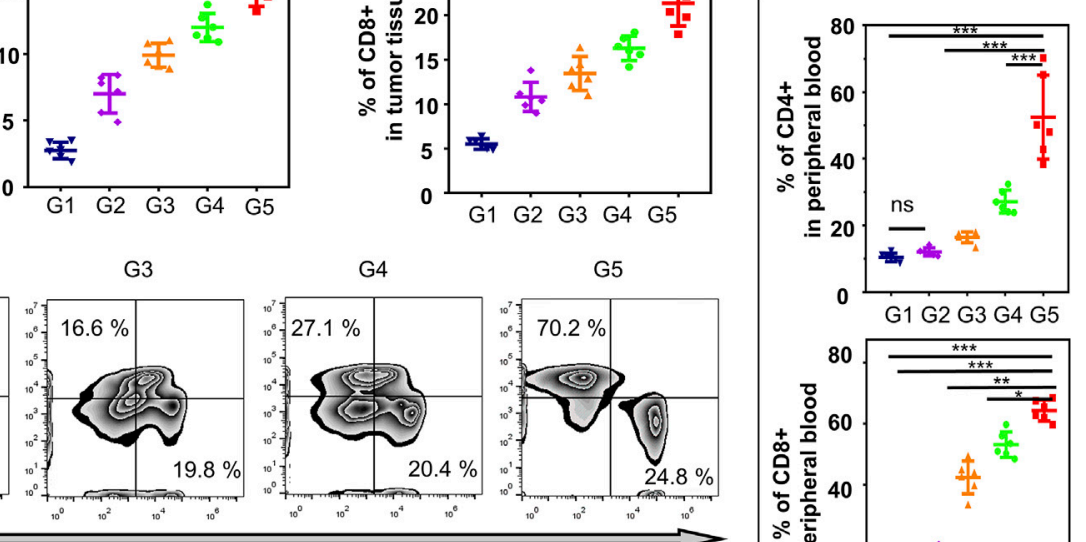

G5

G4

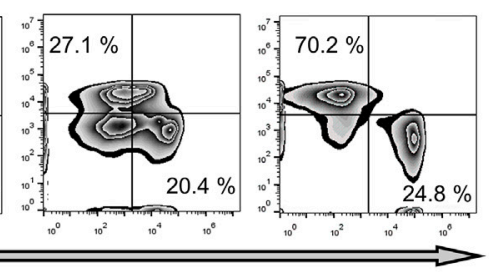

CD8

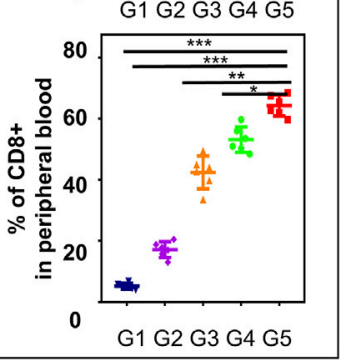

FIGURE 9 | The immune effects from different treatment groups were evaluated in-vivo. (A) Representative immunofluorescence images showing tumor-infiltrating $\mathrm{CD}^{+} \mathrm{CD}^{+}$and $\mathrm{CD}^{+} \mathrm{CD} 8+\mathrm{T}$ cells within $4 \mathrm{~T} 1$ tumors for different treatment groups (G1-G5). (B-D) percentages of $\mathrm{CD} 3^{+}, \mathrm{CD}^{+}$, or $\mathrm{CD} 8^{+} \mathrm{T}$ cells obtained from tumor tissues (A). (E) Representative flow cytometry plots of CTLs $\left(\mathrm{CD4}^{+} \mathrm{CD} 8^{+}\right)$gated on $\mathrm{CD}^{+} \mathrm{CTLs}$ in peripheral blood, respectively. (F) Quantification (\%) of CD4 ${ }^{+} \mathrm{CD} 8^{+}$ gated on $\mathrm{CD}^{+}$CTLs. All data are presented as mean $\pm \mathrm{SD}\left(n=6\right.$, ns, not significant; $\left.{ }^{*} p<0.05,{ }^{\star \star} p<0.01,{ }^{\star \star \star} p<0.001\right)$.

organs (heart, liver, spleen, and kidneys) for all groups, demonstrating that the SCS was safe in vivo.

\section{DISCUSSION}

In the present study, we have combined different therapeutic approaches to treat the most challenging solid cancer, TNBC. Our hypothesis is that to manage high metastasis and high recurrence of TNBC, it is necessary to have a very strong and aggressive treatment. For this, we have designed a "synergistic cascade strategy" based on modifying TME, which consists of a pretreatment of tumor and its surrounding tissue with mild hyperthermia and followed by applying SDDS. The SDDS is composed of two components: 1) intravenous injection of tumor
CD44-targeted thermosensitive liposomes (TSL) preloaded with BML and Ptx, and 2) MW-induced hyperthermia to simultaneously achieve drug release and tumor ablation.

To overcome the resistance of TNBC and to improve the uptake and biodistribution of nanoparticles, the tumor was first irradiated by $\mathrm{MW}$ to induce a mild hyperthermia $\left(43^{\circ} \mathrm{C}\right)$ to alter the TNBC microenvironment. This "hyperthermia-mediated EPR effect" allows to reduce the interstitial pressure of TME and increase intratumoral blood flow and thereby enhance the uptake of nanoparticles at the tumor site. Following the first MW exposure, the nanoparticles HA-BNPs@Ptx was injected into 4T1 tumor-bearing mice via tail vein. Among the hyperthermia techniques, MW heating offers highly competitive advantages: faster heat generation, less susceptibility to heat up local tissues, maneuverability, and 
depth of penetration in tissues (Shi et al., 2015). Although encouraging results are being collected, MW hyperthermia has its own challenges, such as inaccurate targeting and low selectivity, which lead to damage to the surrounding vital organs and tissues (Chen et al., 2017). Therefore, much effort has been devoted to improving the diffusion and the effective accumulation of the heat in the region of the whole tumor by introducing MW-sensitive agents for thermal therapy. In this study, we verified that BML can be used as MW-sensitive agents. BML is a kind of ionic liquid, which has high thermal stability, wide temperature range for the liquid state, low interfacial tension, immeasurable vapor pressure, and high ionic conductivity (Chen et al., 2020). The absorbed microwave energy is transferred to the kinetic and interionic energies of ions and stored as Joule heating energy of salt ions via the interactions between salt ions, which leads to rapid temperature rise (Sorn et al., 2019). The nanoparticles were made of thermosensitive phospholipid DPPC $\left(\mathrm{Tm} \approx 41^{\circ} \mathrm{C}\right)$ and PLGA, which have been extensively used in nanomedicine formulations both for clinical and laboratory for breast cancer treatment. The second $\mathrm{MW}$ irradiation allowed producing a hyper heating effect $\left(\mathrm{T}>50^{\circ} \mathrm{C}\right)$ due to the activation of BML and a rapid release of Ptx from nanoparticles. The increased temperature at the tumor site could directly damage tumor cells and surrounding tissues, inducing apoptosis both in vitro (Figure 4) and in vivo (Figures 7 and 8).

Hyaluronic acid is an important component of the extracellular matrix. It plays an important role in tumor microenvironment and is involved in tumor cell proliferation, invasion, immune escape, stem cell change, and drug resistance (Vasvani et al., 2020). HA is also a ligand for lymphatic vessel endothelial hyaluronan receptor-1 and hyaluronan-mediated motility receptor. HA is a biocompatible, biodegradable, and nonimmunogenic biopolymer capable of actively targeting cluster of CD44 on cell surface receptors overexpressed in many cancer cells, including TNBC (Mattheolabakis et al., 2015; Safdar et al., 2018; Wang et al., 2018; Kari et al., 2020). For all these reasons, we have selected $\mathrm{HA}$ as a ligand to target 4T1 tumor cells that overexpress CD44. Compared with non-targeted nanoparticles, HA-BNPs@Ptx can specifically target TNBC tumor cells both in vitro (Supplementary Figure S4) and in vivo (Figure 5). HA-BNPs@ Ptx nanoparticles exhibited improved cellular uptake, probably via HA receptor-mediated endocytosis and phagocytosis (Figure 4).

In our previous work (Xu et al., 2019a), we have demonstrated that encapsulated BML in P-selectin-targeted nanoparticles could be used to boost the thermal effect of MW-generated hyperthermia and efficiently prevent tumor progression and lung metastasis in HCC tumor-bearing mice. In the present study, we further confirmed that the incorporation of BML in the liposomes can significantly enhance the thermal effect induced by MW irradiation with an increase in temperature of more than $15^{\circ} \mathrm{C}$. It is worth noting that a mild $\mathrm{MW}$ irradiation $\left(0.8 \mathrm{~W} \mathrm{~cm}^{-2}\right.$ and $4 \mathrm{~min}$ ) was sufficient to increase significantly the intratumoral blood flow as shown by CEUS imaging and tumor microvessel density. As a result, the retention of nanoparticles HA-BNPs@Ptx was increased by sevenfold (Figure 6).

Currently, chemotherapy remains the main option for the treatment of TNBC (Oualla et al., 2017; Núñez Abad et al., 2021). However, conventional chemotherapies employed in the treatment of TNBC suffer from issues of poor bioavailability, poor cellular uptake, resistance to drugs, and undesirable off-site toxicities (Bai et al., 2021). Abraxane, a nanoparticle formulation of "Taxol" (paclitaxel), was approved for the treatment of metastatic breast cancer, but the efficacy is limited due to the high heterogeneity and drug resistance of breast tumors (Gradishar, 2006). In the past few years, extensive research has been conducted to overcome drug resistance and improve the prognosis of TNBC (Bai et al., 2021). The combination of chemotherapeutics with SDDS, particularly with stimuliresponsive systems that are able to control drug biodistribution and release in response to specific stimuli, either exogenous (temperature, magnetic field, ultrasound, light, or electric pulses) or endogenous (changes in $\mathrm{pH}$, enzyme concentration, or redox gradients) has been explored for metastatic breast cancer (Rivera-Rodriguez et al., 2018; Yang et al., 2020; Li et al., 2021). The main advantages of combination SDDS with chemotherapy are 1) the decrease in toxicity of chemotherapeutic agents ( $\mathrm{Hu}$ et al., 2020; Thakkar et al., 2020), 2) the local delivery of anticancer agents with higher payloads and optimal distribution (Wang et al., 2021), 3) the use of nanocarriers to deliver immunomodulatory agents that can activate immune cells and modulate TME (Yang and Gao, 2017; Chen et al., 2019; Feng et al., 2020), 4) the incorporation of diagnostic agents for imaging the tumor or TME (Wang et al., 2019; Wang et al., 2020), and 5) the incorporation of active targeting molecules and specific stimuli to have synergistic cascade effects to enhance antitumor efficacy. The results obtained in this study fully demonstrate the virtue of SDDS. Finally, the use of thermal effect to pretreat the tumor and its microenvironment, which allows significantly increasing nanodrug uptake, the so-called "hyperthermia-mediated EPR effect," is an efficient and clinically translatable approach. This approach should be further investigated and explored.

\section{CONCLUSION}

In this study, we demonstrate that mild MW hyperthermia $\left(43^{\circ}-44^{\circ} \mathrm{C}\right)$ can be used to pretreat tumors to promote vasodilation of tumor vessels, improve intratumoral perfusion, and thus increase retention of nanodrugs. HA-BNPs@Ptx as SDDS is highly efficient in active targeting, MW stimulation, and induction of tumor cell apoptosis. In addition, the synergistic cascade effects by combining two-step MW irradiation and HABNPs@Ptx could effectively inhibit progression of triple negative breast tumor in mice. Two-step hyperthermia combined with nanomedicine showed a good synergistic cascade antitumor effect on both solid breast tumor in situ and lung metastasis, thus, providing a new approach for the treatment of breast cancer.

\section{DATA AVAILABILITY STATEMENT}

The original contributions presented in the study are included in the article/Supplementary Material. Further inquiries can be directed to the corresponding authors. 


\section{ETHICS STATEMENT}

The animal study was reviewed and approved by the Chinese Society of Laboratory Animals on animal welfare, The Animal Use and Care Management Advisory Committee of West China Hospital of Sichuan University.

\section{AUTHOR CONTRIBUTIONS}

HZ, JX, and YP designed the study. HZ, BG, HW, JH, JZ, and RY, performed the experiments. HZ, BG, and FY wrote the manuscript. All authors read and approved the final manuscript.

\section{FUNDING}

This research was funded by the Science and Technology Project of Chengdu, (2017-CY02-00027-GX, 2019-YF05-

\section{REFERENCES}

Alam, N., Koul, M., Mintoo, M. J., Khare, V., Gupta, R., Rawat, N., et al. (2017). Development and Characterization of Hyaluronic Acid Modified PLGA Based Nanoparticles for Improved Efficacy of Cisplatin in Solid Tumor. Biomed. Pharmacother. 95, 856-864. doi:10.1016/j.biopha.2017.08.108

Bai, X., Ni, J., Beretov, J., Graham, P., and Li, Y. (2021). Triple-Negative Breast Cancer Therapeutic Resistance: Where Is the Achilles' Heel? Cancer Lett. 497, 100-111. doi:10.1016/j.canlet.2020.10.016

Cerqueira, B. B. S., Lasham, A., Shelling, A. N., and Al-Kassas, R. (2017). Development of Biodegradable PLGA Nanoparticles Surface Engineered with Hyaluronic Acid for Targeted Delivery of Paclitaxel to Triple Negative Breast Cancer Cells. Mater. Sci. Eng. C Mater. Biol. Appl. 76, 593-600. doi:10.1016/j.msec.2017.03.121

Chen, X., Tan, L., Liu, T., and Meng, X. (2017). Micro-Nanomaterials for Tumor Microwave Hyperthermia: Design, Preparation, and Application. Curr. Drug Deliv. 14 (3), 307-322. doi:10.2174/1567201813666160108113805

Chen, Q., Hu, Q., Dukhovlinova, E., Chen, G., Ahn, S., Wang, C., et al. (2019). Photothermal Therapy Promotes Tumor Infiltration and Antitumor Activity of CAR T Cells. Adv. Mater. 31 (23), e1900192. doi:10.1002/adma.201900192

Chen, H., Shen, C., Chen, Z., Hu, J., and Wen, Y. (2020). Disturbance of Chiral Ionic Liquids to Phototaxis of Chlamydomonas Reinhardtii: Regular Analysis and Mechanism Attempt. Environ. Sci. Pollut. Res. Int. 27 (13), 15011-15019. doi:10.1007/s11356-020-07882-6

De Maar, J. S., Suelmann, B. B. M., Braat, M. N. G. J. A., Van Diest, P. J., Vaessen, H. H. B., Witkamp, A. J., et al. (2020). Phase I Feasibility Study of Magnetic Resonance Guided High Intensity Focused Ultrasound-Induced Hyperthermia, Lyso-Thermosensitive Liposomal Doxorubicin and Cyclophosphamide in De Novo Stage IV Breast Cancer Patients: Study Protocol of the I-GO Study. BMJ open 10 (11), e040162. doi:10.1136/ bmjopen-2020-040162

Deepak, K. G. K., Vempati, R., Nagaraju, G. P., Dasari, V. R., S, N., Rao, D. N., et al. (2020). Tumor Microenvironment: Challenges and Opportunities in Targeting Metastasis of Triple Negative Breast Cancer. Pharmacol. Res. 153, 104683. doi:10.1016/j.phrs.2020.104683

Ding, Y., Yang, R., Yu, W., Hu, C., Zhang, Z., Liu, D., et al. (2021). Chitosan Oligosaccharide Decorated Liposomes Combined with $\mathrm{TH} 302$ for Photodynamic Therapy in Triple Negative Breast Cancer. J. Nanobiotechnol 19 (1), 147. doi:10.1186/s12951-021-00891-8

Dou, Y., Hynynen, K., and Allen, C. (2017). To Heat or Not to Heat: Challenges with Clinical Translation of Thermosensitive Liposomes. J. Control. Release 249, 63-73. doi:10.1016/j.jconrel.2017.01.025
00376-SN), Post-Doctor Research Project, West China Hospital, Sichuan University (2020HXBH003), Science and Technology Innovation talent of Sichuan (20CXRC0065), and Science and Technology Project of the Health Planning Committee of Sichuan (20PJ011).

\section{ACKNOWLEDGMENTS}

The authors are grateful to Yan Wang (Research Core Facility of West China Hospital Sichuan university) for her collaboration in Flow cytometer analysis.

\section{SUPPLEMENTARY MATERIAL}

The Supplementary Material for this article can be found online at: https://www.frontiersin.org/articles/10.3389/fphar.2021.750847/ full\#supplementary-material

Dunne, M., Regenold, M., and Allen, C. (2020). Hyperthermia Can Alter Tumor Physiology and Improve Chemo- and Radio-Therapy Efficacy. Adv. Drug Deliv. Rev. 163-4, 98-124. doi:10.1016/j.addr.2020.07.007

Feng, C., Xiong, Z., Wang, C., Xiao, W., Xiao, H., Xie, K., et al. (2020). Folic AcidModified Exosome-PH20 Enhances the Efficiency of Therapy via Modulation of the Tumor Microenvironment and Directly Inhibits Tumor Cell Metastasis. Bioact Mater. 6 (4), 963-974. doi:10.1016/j.bioactmat.2020.09.014

Furman, C., Carpentier, R., Barczyk, A., Chavatte, P., Betbeder, D., and Lipka, E. (2017). Development and Validation of a Reversed-phase HPLC Method for the Quantification of Paclitaxel in Different PLGA Nanocarriers. Electrophoresis 38 (19), 2536-2541. doi:10.1002/elps.201600552

Gradishar, W. J. (2006). Albumin-Bound Paclitaxel: a Next-Generation Taxane. Expert Opin. Pharmacother. 7 (8), 1041-1053. doi:10.1517/14656566.7.8.1041

Griffon-Etienne, G., Boucher, Y., Brekken, C., Suit, H. D., and Jain, R. K. (1999). Taxane-induced Apoptosis Decompresses Blood Vessels and Lowers Interstitial Fluid Pressure in Solid Tumors: Clinical Implications. Cancer Res. 59 (15), 3776-3782.

Hu, Q., Shang, L., Wang, M., Tu, K., Hu, M., Yu, Y., et al. (2020). Co-Delivery of Paclitaxel and Interleukin-12 Regulating Tumor Microenvironment for Cancer Immunochemotherapy. Adv. Healthc. Mater. 9 (10), e1901858. doi:10.1002/ adhm.201901858

Hynynen, K. (2018). Hyperthermia-induced Drug Delivery in Humans. Nat. Biomed. Eng. 2 (9), 637-639. doi:10.1038/s41551-018-0297-8

Januškevičienè, I., and Petrikaitė, V. (2019). Heterogeneity of Breast Cancer: The Importance of Interaction between Different Tumor Cell Populations. Life Sci. 239, 117009. doi:10.1016/j.lfs.2019.117009

Junttila, M. R., and de Sauvage, F. J. (2013). Influence of Tumour Microenvironment Heterogeneity on Therapeutic Response. Nature 501 (7467), 346-354. doi:10.1038/nature12626

Kari, O. K., Tavakoli, S., Parkkila, P., Baan, S., Savolainen, R., Ruoslahti, T., et al. (2020). Light-Activated Liposomes Coated with Hyaluronic Acid as a Potential Drug Delivery System. Pharmaceutics 12 (8), 763 . doi:10.3390/ pharmaceutics12080763

Lang, T., Liu, Y., Zheng, Z., Ran, W., Zhai, Y., Yin, Q., et al. (2019). Cocktail Strategy Based on Spatio-Temporally Controlled Nano Device Improves Therapy of Breast Cancer. Adv. Mater. 31 (5), e1903844. doi:10.1002/ adma.201806202

Li, Q., Yang, J., Chen, C., Lin, X., Zhou, M., Zhou, Z., et al. (2020). A Novel Mitochondrial Targeted Hybrid Peptide Modified HPMA Copolymers for Breast Cancer Metastasis Suppression. J. Control. Release 325, 38-51. doi:10.1016/j.jconrel.2020.06.010

Li, X., Jeon, Y. H., Kwon, N., Park, J. G., Guo, T., Kim, H. R., et al. (2021). In VivoAssembled Phthalocyanine/albumin Supramolecular Complexes Combined 
with a Hypoxia-Activated Prodrug for Enhanced Photodynamic Immunotherapy of Cancer. Biomaterials 266, 120430. doi:10.1016/ j.biomaterials.2020.120430

Lyon, P. C., Gray, M. D., Mannaris, C., Folkes, L. K., Stratford, M., Campo, L., et al. (2018). Safety and Feasibility of Ultrasound-Triggered Targeted Drug Delivery of Doxorubicin from Thermosensitive Liposomes in Liver Tumours (TARDOX): a single-centre, Open-Label, Phase 1 Trial. Lancet Oncol. 19 (8), 1027-1039. doi:10.1016/S1470-2045(18)30332-2

Markezana, A., Ahmed, M., Kumar, G., Zorde-Khvalevsky, E., Rozenblum, N., Galun, E., et al. (2020). Moderate Hyperthermic Heating Encountered during thermal Ablation Increases Tumor Cell Activity. Int. J. Hyperthermia 37 (1), 119-129. doi:10.1080/02656736.2020.1714084

Mattheolabakis, G., Milane, L., Singh, A., and Amiji, M. M. (2015). Hyaluronic Acid Targeting of CD44 for Cancer Therapy: from Receptor Biology to Nanomedicine. J. Drug Target. 23 (7-8), 605-618. doi:10.3109/ 1061186X.2015.1052072

May, J. P., and Li, S. D. (2013). Hyperthermia-induced Drug Targeting. Expert Opin. Drug Deliv. 10 (4), 511-527. doi:10.1517/17425247.2013.758631

$\mathrm{Mu}$, Q., Wang, H., and Zhang, M. (2017). Nanoparticles for Imaging and Treatment of Metastatic Breast Cancer. Expert Opin. Drug Deliv. 14 (1), 123-136. doi:10.1080/17425247.2016.1208650

Nardecchia, S., Sánchez-Moreno, P., Vicente, J., Marchal, J. A., and Boulaiz, H. (2019). Clinical Trials of Thermosensitive Nanomaterials: An Overview. Nanomaterials (Basel) 9 (2), 191. doi:10.3390/nano9020191

Nave, M., Castro, R. E., Rodrigues, C. M., Casini, A., Soveral, G., and Gaspar, M. M. (2016). Nanoformulations of a Potent Copper-Based Aquaporin Inhibitor with Cytotoxic Effect against Cancer Cells. Nanomedicine (Lond) 11 (14), 1817-1830. doi:10.2217/nnm-2016-0086

Nia, H. T., Munn, L. L., and Jain, R. K. (2020). Physical Traits of Cancer. Science 370 (6516), eaaz0868. doi:10.1126/science.aaz0868

Nienhuis, H. H., Gaykema, S. B., Timmer-Bosscha, H., Jalving, M., Brouwers, A. H., Lub-de Hooge, M. N., et al. (2015). Targeting Breast Cancer through its Microenvironment: Current Status of Preclinical and Clinical Research in Finding Relevant Targets. Pharmacol. Ther. 147, 63-79. doi:10.1016/ j.pharmthera.2014.11.004

Núñez Abad, M., Calabuig-Fariñas, S., Lobo de Mena, M., José Godes Sanz de Bremond, M., García González, C., Torres Martínez, S., et al. (2021). Update on Systemic Treatment in Early Triple Negative Breast Cancer. Ther. Adv. Med. Oncol. 13, 175883592098674. doi:10.1177/1758835920986749

Oualla, K., El-Zawahry, H. M., Arun, B., Reuben, J. M., Woodward, W. A., Gamal El-Din, H., et al. (2017). Novel Therapeutic Strategies in the Treatment of Triple-Negative Breast Cancer. Ther. Adv. Med. Oncol. 9 (7), 493-511. doi:10.1177/1758834017711380

Pan, J., Xu, Y., Wu, Q., Hu, P., and Shi, J. (2021). Mild Magnetic HyperthermiaActivated Innate Immunity for Liver Cancer Therapy. J. Am. Chem. Soc. 143 (21), 8116-8128. doi:10.1021/jacs.1c02537

Pashayan, N., Antoniou, A. C., Ivanus, U., Esserman, L. J., Easton, D. F., French, D., et al. (2020). Personalized Early Detection and Prevention of Breast Cancer: ENVISION Consensus Statement. Nat. Rev. Clin. Oncol. 17 (11), 687-705. doi:10.1038/s41571-020-0388-9

Paulides, M. M., Dobsicek Trefna, H., Curto, S., and Rodrigues, D. B. (2020). Recent Technological Advancements in Radiofrequency- AndmicrowaveMediated Hyperthermia for Enhancing Drug Delivery. Adv. Drug Deliv. Rev. 163-164, 3-18. doi:10.1016/j.addr.2020.03.004

Qi, J., Li, W., Lu, K., Jin, F., Liu, D., Xu, X., et al. (2019). pH and Thermal DualSensitive Nanoparticle-Mediated Synergistic Antitumor Effect of Immunotherapy and Microwave Thermotherapy. Nano Lett. 19 (8), 4949-4959. doi:10.1021/acs.nanolett.9b01061

Rivera-Rodriguez, A., Chiu-Lam, A., Morozov, V. M., Ishov, A. M., and Rinaldi, C. (2018). Magnetic Nanoparticle Hyperthermia Potentiates Paclitaxel Activity in Sensitive and Resistant Breast Cancer Cells. Int. J. Nanomed. 13, 4771-4779. doi:10.2147/IJN.S171130

Safdar, M. H., Hussain, Z., Abourehab, M. A. S., Hasan, H., Afzal, S., and Thu, H. E. (2018). New Developments and Clinical Transition of Hyaluronic AcidBased Nanotherapeutics for Treatment of Cancer: Reversing Multidrug Resistance, Tumour-specific Targetability and Improved Anticancer Efficacy. Artif. Cell Nanomed. Biotechnol. 46 (8), 1967-1980. doi:10.1080/ 21691401.2017.1397001
Seynhaeve, A. L. B., Amin, M., Haemmerich, D., Van Rhoon, G. C., and Ten Hagen, T. L. M. (2020). Hyperthermia and Smart Drug Delivery Systems for Solid Tumor Therapy. Adv. Drug Deliv. Rev. 163-164, 125-144. doi:10.1016/ j.addr.2020.02.004

Shi, H., Liu, T., Fu, C., Li, L., Tan, L., Wang, J., et al. (2015). Insights into a Microwave Susceptible Agent for Minimally Invasive Microwave Tumor thermal Therapy. Biomaterials 44, 91-102. doi:10.1016/ j.biomaterials.2014.12.035

Sorn, V., Chang, K. L., Phitsuwan, P., Ratanakhanokchai, K., and Dong, C. D. (2019). Effect of Microwave-Assisted Ionic Liquid/acidic Ionic Liquid Pretreatment on the Morphology, Structure, and Enhanced Delignification of rice Straw. Bioresour. Technol. 293, 121929. doi:10.1016/ j.biortech.2019.121929

Sun, W., Du, Y., Liang, X., Yu, C., Fang, J., Lu, W., et al. (2019). Synergistic TripleCombination Therapy with Hyaluronic Acid-Shelled PPy/CPT Nanoparticles Results in Tumor Regression and Prevents Tumor Recurrence and Metastasis in 4T1 Breast Cancer. Biomaterials 217, 119264. doi:10.1016/ j.biomaterials.2019.119264

Tajbakhsh, A., Hasanzadeh, M., Rezaee, M., Khedri, M., Khazaei, M., ShahidSales, S., et al. (2018). Therapeutic Potential of Novel Formulated Forms of Curcumin in the Treatment of Breast Cancer by the Targeting of Cellular and Physiological Dysregulated Pathways. J. Cel Physiol 233 (3), 2183-2192. doi:10.1002/jcp.25961

Tak, W. Y., Lin, S. M., Wang, Y., Zheng, J., Vecchione, A., Park, S. Y., et al. (2018). Phase III HEAT Study Adding Lyso-Thermosensitive Liposomal Doxorubicin to Radiofrequency Ablation in Patients with Unresectable Hepatocellular Carcinoma Lesions. Clin. Cancer Res. 24 (1), 73-83. doi:10.1158/10780432.CCR-16-2433

Thakkar, S., Sharma, D., Kalia, K., and Tekade, R. K. (2020). Tumor Microenvironment Targeted Nanotherapeutics for Cancer Therapy and Diagnosis: A Review. Acta Biomater. 101, 43-68. doi:10.1016/j.actbio.2019.09.009

Thakur, V., and Kutty, R. V. (2019). Recent Advances in Nanotheranostics for Triple Negative Breast Cancer Treatment. J. Exp. Clin. Cancer Res. 38 (1), 430. doi:10.1186/s13046-019-1443-1

Vasvani, S., Kulkarni, P., and Rawtani, D. (2020). Hyaluronic Acid: A Review on its Biology, Aspects of Drug Delivery, Route of Administrations and a Special Emphasis on its Approved Marketed Products and Recent Clinical Studies. Int. J. Biol. Macromol 151, 1012-1029. doi:10.1016/j.ijbiomac.2019.11.066

Wang, Z., Sau, S., Alsaab, H. O., and Iyer, A. K. (2018). CD44 Directed Nanomicellar Payload Delivery Platform for Selective Anticancer Effect and Tumor Specific Imaging of Triple Negative Breast Cancer. Nanomedicine 14 (4), 1441-1454. doi:10.1016/j.nano.2018.04.004

Wang, Z., Ju, Y., Ali, Z., Yin, H., Sheng, F., Lin, J., et al. (2019). Near-infrared Light and Tumor Microenvironment Dual Responsive Size-Switchable Nanocapsules for Multimodal Tumor Theranostics. Nat. Commun. 10 (1), 4418. doi:10.1038/ s41467-019-12142-4

Wang, Z., Li, Z., Sun, Z., Wang, S., Ali, Z., Zhu, S., et al. (2020). Visualization Nanozyme Based on Tumor Microenvironment "Unlocking" for Intensive Combination Therapy of Breast Cancer. Sci. Adv. 6 (48), eabc8733. doi:10.1126/sciadv.abc8733

Wang, Y., Zhang, Z., Zheng, C., Zhao, X., Zheng, Y., Liu, Q., et al. (2021). Multistage Adaptive Nanoparticle Overcomes Biological Barriers for Effective Chemotherapy. Small 17, 2100578. doi:10.1002/smll.202100578

Wu, Q., Xia, N., Long, D., Tan, L., Rao, W., Yu, J., et al. (2019). Dual-Functional Supernanoparticles with Microwave Dynamic Therapy and Microwave Thermal Therapy. Nano Lett. 19 (8), 5277-5286. doi:10.1021/ acs.nanolett.9b01735

Xu, J., Cheng, X., Tan, L., Fu, C., Ahmed, M., Tian, J., et al. (2019). Microwave Responsive Nanoplatform via P-Selectin Mediated Drug Delivery for Treatment of Hepatocellular Carcinoma with Distant Metastasis. Nano Lett. 19 (5), 2914-2927. doi:10.1021/acs.nanolett.8b05202

Xu, J., Liu, Y., Li, Y., Wang, H., Stewart, S., Van der Jeught, K., et al. (2019). Precise Targeting of POLR2A as a Therapeutic Strategy for Human Triple Negative Breast Cancer. Nat. Nanotechnol 14 (4), 388-397. doi:10.1038/s41565-0190381-6

Yang, S., and Gao, H. (2017). Nanoparticles for Modulating Tumor Microenvironment to Improve Drug Delivery and Tumor Therapy. Pharmacol. Res. 126, 97-108. doi:10.1016/j.phrs.2017.05.004 
Yang, Y., Qiao, X., Huang, R., Chen, H., Shi, X., Wang, J., et al. (2020). E-jet 3D Printed Drug Delivery Implants to Inhibit Growth and Metastasis of Orthotopic Breast Cancer. Biomaterials 230, 119618. doi:10.1016/ j.biomaterials10.1016/j.biomaterials.2019.119618

Zhang, Y., Hu, H., Tang, W., Zhang, Q., Li, M., Jin, H., et al. (2020). A Multifunctional Magnetic Nanosystem Based on "two Strikes" Effect for Synergistic Anticancer Therapy in Triple-Negative Breast Cancer. J. Control. Release 322, 401-415. doi:10.1016/ j.jconrel.2020.03.036

Conflict of Interest: The authors declare that the research was conducted in the absence of any commercial or financial relationships that could be construed as a potential conflict of interest.
Publisher's Note: All claims expressed in this article are solely those of the authors and do not necessarily represent those of their affiliated organizations, or those of the publisher, the editors, and the reviewers. Any product that may be evaluated in this article, or claim that may be made by its manufacturer, is not guaranteed or endorsed by the publisher.

Copyright (c) 2021 Zhang, Xu, Gao, Wang, Huang, Zhou, Yang, Yan and Peng. This is an open-access article distributed under the terms of the Creative Commons Attribution License (CC BY). The use, distribution or reproduction in other forums is permitted, provided the original author(s) and the copyright owner(s) are credited and that the original publication in this journal is cited, in accordance with accepted academic practice. No use, distribution or reproduction is permitted which does not comply with these terms. 